\title{
Lecture, répétition, parole spontanée : l'impact de la tâche sur le comportement du schwa en FLE
}

\author{
Julia Kamerhuber, Julia Horvath et Elissa Pustka \\ Université de Vienne \\ Email: elissa.pustka@univie.ac.at
}

(Received 09 May 2019; revised 21 January 2020; accepted 22 January 2020)

\begin{abstract}
RESUME
L'objectif de cet article est de déterminer l'impact de la tâche sur la prononciation du FLE, en l'occurrence sur le comportement du schwa. Dans cette perspective sont étudiées les productions de 145 apprenants autrichiens du FLE de niveau débutant à B1 dans la lecture d'une liste de mots et de deux textes, dans une tâche de répétition de mots ainsi que dans un entretien guidé (corpus de 87 heures et 45 minutes). L'étude se concentre sur la production du schwa dans huit mots et constructions : s(e)maine, ch(e)mise, premier, $m$ (on)sieur, tout l(e) temps, tout l(e) monde, appartement et gouvernement. Nos résultats montrent que les 35 heures de parole spontanée ne contiennent que peu ou même pas d'occurrences de ces mots pour la moitié des cas analysés. Bien qu'il s'agisse d'une méthode valable pour l'analyse du système linguistique, il s'avère dans la recherche de la prononciation des apprenants indispensable de la compléter par des tâches d'élicitation. L'élicitation par des formes graphiques présente pourtant l'inconvénient d'influencer la prononciation par la présence visuelle des lettres (sans différence notable entre la liste de mots et les textes). Cette influence ne se limite pas au processus de décodage en temps réel.
\end{abstract}

\section{INTRODUCTION}

L'un des grands défis de la recherche sur l'acquisition et l'apprentissage des langues secondes est le problème d'ordre très général de trouver le juste équilibre entre la comparabilité des données et leur validité écologique. Dans le domaine de la prononciation s'opposent ainsi des méthodes d'élicitation telles que la lecture, la répétition, la dénomination d'images et des méthodes de recueil de parole plus spontanée, comme l'entretien ( $c f$. Shea, 2013 ; Munro et Derwing, 2015).

\footnotetext{
Nous remercions le FWF (Fonds autrichien de la recherche scientifique) pour le financement du projet Pro $^{2} \mathrm{~F}$, notre lycée partenaire (qui doit rester anonyme) pour sa collaboration, les étudiantes-assistantes (Daphné Baudry, Julia Gusterer, Johanna Himmer, Léonore Troehler, Barbara Huber, Romana Schneider, Linda Schwärzler, Angelika Steinringer et Magdalena Ulleram) pour leur travail de terrain, les transcriptions et les codages et finalement Marc Chalier pour les enregistrements du locuteur-modèle et la relecture de ce texte.

(c) Cambridge University Press 2020. This is an Open Access article, distributed under the terms of the Creative Commons Attribution licence (http://creativecommons.org/licenses/by/4.0/), which permits unrestricted re-use, distribution, and reproduction in any medium, provided the original work is properly cited.
} 
Dans le cadre du présent article, nous comparons les données obtenues grâce à quatre tâches, dont trois tâches d'élicitation (lecture d'une liste de mots, lecture d'un texte, répétition de mots polysyllabiques) et une tâche de parole spontanée (entretien guidé). L'objet de notre analyse est le schwa, variable phonologique encore peu étudiée en français langue étrangère (FLE) ( $c f$. section 2.2) et peu ou pas traité dans l'enseignement (cf. section 3.1). Bien qu'il existe aussi un $<\mathrm{e}>$ correspondant à schwa en allemand, son articulation phonétique, les correspondances graphie-phonie dans une bonne partie des contextes ainsi que son comportement phonologique ne sont pas les mêmes. On devrait donc s'attendre à des problèmes très complexes dans ce domaine.

Nous nous concentrons sur deux positions phonotactiques en particulier : la première syllabe des mots polysyllabiques et la syllabe interne de mots polysyllabiques et de constructions polysyllabiques. Ce faisant, notre étude est centrée sur la production du schwa dans les huit mots et constructions suivants : s(e)maine, ch(e)mise, premier, $m(\underline{o n})$ sieur, tout l(e) temps, tout l(e) monde, appartement et gouvernement. Le choix de ces mots a été guidé par trois facteurs : leur fréquence supposée en classe (basé sur la première occurrence et leur fréquence dans les manuels scolaires ; cf. Tableau 3$)$, les différents rapports phonème-graphème $(<\mathrm{e}>$ vs $<\mathrm{on}>$ dans monsieur) ainsi que l'influence de la L1/première langue étrangère (dans les cas de premier, appartement et gouvernement). De plus, nous avons donné la préférence aux mots déjà connus par nos élèves (suivant les manuels scolaires).

D’après les ouvrages normatifs ( $c f$. Fouché, 1959 ; Léon, 2011), le schwa se comporte de la manière suivante en français L1 :

- le schwa est toujours prononcé après deux consonnes (p.ex. premier, appartement) ;

- le schwa est élidé en syllabe interne après une seule consonne (p.ex. tout l(e) monde, tout l(e) temps), et en finale de mot (contexte non étudié ici) ;

- le schwa est variable en première syllabe de polysyllabes (p.ex. s(e)maine).

Les études empiriques sur le français parisien, souvent considéré comme 'standard'1 confirment ces tendances (cf. Hansen, 1994 ; Pustka, 2007 ; Lyche et Østby, 2009). Nous faisons abstraction ici, d'une part, des schwas internes après une seule consonne et des schwas finaux, qui, conformément à la norme de prononciation $\mathrm{du}$ français, sont quasiment toujours élidés par les apprenants de notre corpus et, d'autre part, des schwas dans les clitiques, qui sont quasiment toujours prononcés bien qu'ils soient variables en français L1 (à l'exception des clitiques dans les deux constructions figées tout l(e) temps et tout l(e) monde dans lesquelles ils sont toujours élidés par les locuteurs L1 du français). Les données ont été récoltées dans le cadre du projet Pro $^{2} \mathrm{~F}$ (Pronunciation in Progress : French Schwa and Liaison) basé sur le cadre méthodologique du programme (I)PFC.

La comparaison des tâches permet de conclure qu'une bonne partie des erreurs remonte au fait que les élèves n'apprennent ni les correspondances graphie-phonie,

\footnotetext{
${ }^{1}$ Pour une discussion in extenso de la question de la norme de prononciation du français (ainsi que des termes français standard et français de référence) cf. Chalier (2019).
} 
ni les règles phonologiques du français ni la prononciation des mots individuels, mais qu'ils acquièrent leurs représentations cognitives sur la base des formes graphiques ainsi que de leurs représentations des cognats dans leur L1 et leur première langue étrangère, l'anglais.

\section{2. ÉTAT DE LA QUESTION}

\subsection{Méthodes de recueil des données phonologiques}

Toute étude linguistique est confrontée au problème du choix des méthodes de recueil. Étant donné le paradoxe de l'observateur (cf. Labov, 1972 : 209), il est impossible d'enregistrer de façon systématique l'usage de locuteurs tel qu'il serait observable si ces locuteurs n'étaient pas enregistrés. Le chercheur se trouve donc placé devant un choix entre validité et comparabilité : alors que la parole spontanée s'approche davantage d'une validité écologique, les tâches de lecture ou de répétition ont l'avantage d'être plus comparables (tous les locuteurs produisant les mêmes mots). Dans le sens inverse, la parole spontanée présente l'inconvénient de ne pas pouvoir permettre un contrôle du choix des lexèmes utilisés par les locuteurs. Quant à la lecture, la forme graphique peut influencer la prononciation (effet Buben d'après Buben, 1935) et mener notamment à la réalisation de sons qui ne seraient pas produits en parole spontanée. De plus, les participants, qu'ils lisent des listes de mots sans contextes, ou un texte, ont tendance à se concentrer fortement sur la prononciation (audio-monitoring ; Labov, 1972 : 208). Dans les études sur le français, l'influence de la graphie joue un rôle particulièrement important car l'acquisition du rapport graphèmephonème est un des défis majeurs pour les apprenants du français langue étrangère.

Dans la recherche sur la prononciation des langues étrangères, afin de contourner l'influence du facteur tâche sur le résultat, on utilise souvent la dénomination d'images et la répétition ( $c f$. Moyer, 2007 ; Munro et Derwing, 2015 ; Shea, 2013). Si la première ne se prête cependant qu'à un nombre limité de noms concrets, et la seconde permet de révéler les compétences théoriques des locuteurs ('to discover whether a learner can "sound native" ', Moyer, 2007 : 113 ; souligné par les auteures), les deux tâches se trouvent critiquée pour leur manque de validité :

The merit of highly controlled tasks such as picture naming and repetition is that they guarantee similar speech material from all speakers, which is necessary if direct comparisons are to be made. On the other hand, the trade-off between control and ecological validity is also pertinent here, since uncontrolled tasks (e.g., unrehearsed narratives) are generally more representative of real world speech. (Munro et Derwing, 2015:24; mise en relief par les auteures de cet article)

De plus, la tâche de répétition soulève la question de savoir quel type de locuteur pourrait être concrètement considéré comme modèle quant à son usage. Alors que de nombreux chercheurs considèrent la norme comme une construction artificielle (cf. Morin, 2000), Detey et Le Gac (2008), Detey et al. (2016) et Chalier (2018) proposent de la fonder sur des études empiriques étudiant les représentations et 
perceptions des locuteurs-auditeurs non-experts. Chalier (2019) montre pour Paris, la Suisse romande et le Québec que les présentateurs de radio et de télévision sont majoritairement considérés comme représentants du français standard.

Moyer (2007), Shea (2013) ainsi que Munro et Derwing, (2015) recommandent une triangulation de plusieurs méthodes afin d'obtenir des données comparables et valables. C'est la solution qui avait déjà été avancée dans le cadre de la sociolinguistique labovienne, qui combinait la lecture de paires minimales, d'une liste de mots, d'un texte et de la parole spontanée ( $c f$. Labov, 1972). Une telle combinaison de méthodes est intéressante dans une perspective sociolinguistique, mais également du point de vue de l'économie de la recherche. D'un côté, les réalisations apparaissant exclusivement ou plus fréquemment dans les tâches plus contrôlées nous donnent des indications par rapport à la représentation et aux attitudes des locuteurs et, plus particulièrement, à leur rapport à la norme. D'un autre côté, une opposition phonémique qui n'est pas faite dans les tâches les plus contrôlées n'apparaîtra pas non plus dans les tâches moins contrôlées, ce qui facilite les analyses (cf. Detey et al., $2016: 15$ ).

Ce choix méthodologique a été repris par le programme de recherche Phonologie $d u$ Français Contemporain (PFC ; www.projet-PFC.net, Durand, Laks et Lyche, 2002) et par son sous-projet Interphonologie du Français Contemporain (IPFC), qui porte sur la prononciation des apprenants de français L2 (cf. http://cblle.tufs. ac.jp/ipfc/ ; Racine et al., 2012). Le programme PFC compare la lecture de paires minimales avec la lecture de mots dans une liste en ordre aléatoire, la lecture d'un texte et deux types de parole spontanée (entretien guidé et discussion libre). L'expérience montre cependant qu'entretien et discussion n'apportent guère de résultats différents ( $c f$. les études dans Durand, Laks et Lyche, 2009 ; Detey et al., 2016). Si on retourne au schwa, l'opposition entre lecture et parole spontanée, en revanche, est remarquable. Pour les 143 locuteurs de 13 enquêtes menées en France septentrionale, Lyche (2016) reporte les résultats suivants : dans les clitiques, après une seule consonne, les schwas sont réalisés dans $35 \%$ des cas (2695/7786) en parole spontanée et, selon la tranche d'âge, entre 88,3\% (26-59 ans) et $94,5 \%$ ( $<26$ ans) des cas en lecture ( $91,3 \%$ chez les plus de 59 ans). En première syllabe de polysyllabes, $34 \%$ des schwas $(658 / 1925)$ sont réalisés en parole spontanée contre $95,7 \%$ ( $>59$ ans) et $100 \%$ ( $<26$ ans) en lecture. ${ }^{2}$

IPFC remplace la discussion libre par une interaction entre apprenants basée sur des instructions et ajoute une tâche de répétition. L'analyse des voyelles $[\mathrm{u}]$ et $[\mathrm{y}]$ chez les apprenants hispanophones par Racine et Detey (2019) montre pour ce phénomène que la prononciation des apprenants est plus proche de la norme du français L1 dans la tâche de répétition que dans la tâche de lecture, ce qui suggère que la forme graphique possède un effet perturbant.

\subsection{Schwa en FLE}

Jusqu'ici, la prononciation ou non du schwa par les apprenants reste encore peu étudiée en FLE surtout au niveau débutant, la plupart des études existantes

\footnotetext{
${ }^{2}$ Il s'agit de nos propres calculs effectués sur la base des données de Lyche (2016). Pour la parole spontanée, elle donne des chiffres absolus, pour la lecture uniquement des pourcentages.
} 
se limitant aux apprenants intermédiaires et avancés (à partir du niveau B1 du CECR, ${ }^{3}$ notamment apprenants de français de niveau universitaire). L'apprentissage du schwa chez les débutants en milieu scolaire n'a, pour sa part, encore été sujet d'aucune enquête jusqu'à présent. Deux études ont été réalisées au Canada (Thomas, 2001, 2002, 2004 ; Uritescu et al., 2002, 2004 ${ }^{4}$ ), où la norme cible et, en particulier, le comportement du schwa sont bien différents de ce qui peut être observé en France (la majorité des schwas étant élidés, pour plus de détails $c f$. Côté, 2012). Les apprenants analysés ont l'anglais comme L1. Plusieurs enquêtes ont été menées dans le cadre du projet IPFC ( $c f$. section 2.1), entre autres des sous-projets du programme IPFC-allemand, étudiant des locuteurs du Nord et du Sud de l'Allemagne (Pustka et Meisenburg, 2017), de l'Autriche (Pustka, Forster et Kamerhuber, 2018) et de la Suisse alémanique (Isely et al., 2018). Ces enquêtes seront présentées dans ce qui suit.

Uritescu et al. (2002) et (2004) comparent la prononciation de 8 élèves canadiens provenant de Toronto, ayant l'anglais comme L1 et apprenant le français dans le cadre d'un programme d'immersion, à celle de 5 (2002) et ensuite 9 (2004) élèves ontariens de la région de North Bay. Ils y étudient le schwa en lecture et en entretien, entre autres dans les quatre contextes nous intéressant ici : en première syllabe et en syllabe interne de polysyllabe, après une et deux consonnes. De manière générale, l'étude montre que les apprenants avancés élident moins de schwas que les locuteurs L1 du français. En ce qui concerne la différence entre les tâches, les chercheurs concluent que la lecture ne diffère guère de la parole spontanée.

Quant aux contextes internes qui sont analysés dans cet article, les études d'Uritescu et al. (2002) et (2004) fournissent les résultats suivants : en entretien, le schwa est élidé dans $8,84 \%(16 / 181)$ des cas en première syllabe après une seule consonne (p. ex. il va $v($ e)nir) et dans $9 \%$ des cas après deux consonnes (p. ex. sept semaines) ; en syllabe interne, il l'est dans $78,11 \%$ des cas après une seule consonne (p. ex. maint(e)nant) et dans $80 \%$ des cas après deux consonnes (p. ex. exactement). Ce dernier résultat, qui est particulièrement surprenant à première vue, car le schwa y est normalement maintenu par les locuteurs L1 du français, s'explique par l'influence des équivalents anglais (cognats), notamment angl. government pour fr. gouvernement et angl. apartment pour fr. appartement ${ }^{6}$. Pour la lecture, l'étude ne fournit qu'un pourcentage d'élision pour la première syllabe après une consonne : $15,78 \%$ (3/19). Il est donc assez surprenant que le taux d'élision du schwa soit plus élevé en lecture qu'en entretien $(8,84 \%, 16 / 181$; cf. supra), mais cela pourrait être un artéfact du nombre total d'occurrences peu élevé en lecture.

Une deuxième enquête canadienne est présentée dans les mêmes années par Thomas (2001), (2002) (en français) et (2004) (en anglais). Elle se base sur 12 minutes de parole

\footnotetext{
${ }^{3} C f$. Conseil de l'Europe 2018.

${ }^{4}$ Ces premières études ne présentent pas encore le niveau de compétence en fonction du CECR qui n'a été publié qu'en 2001.

${ }^{5}$ Il s'agit de deux publications de la même étude, 2002 en français et 2004 en anglais.

${ }^{6}$ Selon les règles normatives, le schwa après deux consonnes est obligatoire, comme dans appartement. Lyche et Østby (2009 : 216) montrent cependant qu'il existe aussi des exceptions : en parole spontanée, dans le corpus PFC, 5 des 41 occurrences qu'elles relèvent présentent en effet un schwa élidé dans ce contexte. Les locuteurs proviennent majoritairement de Lyon et de Paris.
} 
spontanée ainsi que sur la lecture d'un texte par 87 étudiants ontariens. En parole spontanée, l'analyse se concentre sur le schwa se trouvant après une seule consonne, en position interne de polysyllabe et en monosyllabe. Les taux de maintien du schwa dans le corpus atteignent $17 \%$ en contexte $\mathrm{VC@C} \mathrm{C}^{7}$ et $77,9 \%$ en contexte V\#C@\#C (et même 99,9\% en lecture). Thomas (2002:7) observe une variation lexicale importante (en lecture) : chang(e)ment : $0 \%$ de maintien du schwa, jug(e) ments : $0 \%$, certain(e)ment : $2,3 \%$, boul(e)versement : $9,8 \%$, enn(e)mis : $58 \%$. Pour ennemis, l'auteur avance l'explication du cognat anglais enemy. Dans les autres cas, le suffixe -ment favoriserait l'élision du schwa précédent. De plus, 90,2\% des schwas sont maintenus dans la construction figée $c(\underline{e}) q u i$, alors que le taux d'élision s'élève à $99,9 \%$ dans les autres cas du même contexte.

Dans le cadre du programme de recherche IPFC ( $c f$. section 2.2), plusieurs enquêtes sur les apprenants ont récemment été exploitées pour le schwa. Pustka et al. (2017) se basent sur des enregistrements d'étudiants de français d'Osnabrück (6 locuteurs de niveau A2/B1 et 6 de niveau C1), dans le Nord de l'Allemagne, et de Munich (12 locuteurs de niveau B1 à C2), dans le Sud. Elles notent que les locuteurs réalisent souvent une voyelle erronée $[\mathrm{e}]$ ou $[\varepsilon]$ au lieu d'un schwa [ə], p. ex. dans les mots depuis avec [e] et indiqu(e)raient avec [e]. Elles expliquent ces réalisations par une interférence de l'allemand, où le schwa possède des variantes hyperarticulées, [e] et $[\varepsilon]$ (p.ex. dans Muskel [mus.kel] au lieu de ['mus.kl]. Pour des apprenants germanophones, le schwa français ne demande pourtant pas l'acquisition d'une nouvelle voyelle ( $c f$. Flege, 1987) car l'allemand connait aussi [ø] ou [œ] qui sont les réalisions communes du schwa français (cf. Detey et al., 2010 ; Fougeron, Gendrot et Bürki, 2007). Pustka, Forster et Kamerhuber (2018) présentent les résultats du corpus IPFC-Vienne (6 locuteurs de niveau A2/B1, 6 de niveau $\mathrm{C} 1$ ). Les problèmes observés ici sont, d'une part, l'absence d'élision dans $67 \%$ des cas en position interne après une seule consonne (p. ex. bêt(e)ment) et, d'autre part, la réalisation de $[\mathrm{e}]$ ou $[\varepsilon]$ au lieu de [ə]. De plus, ce corpus présente des non-réalisations dans gouvernement, qui s'expliquent à nouveau par le cognat anglais (cf. supra), et des prononciations de récemment comme [веsəmã] au lieu de [веsamã], dû à la méconnaissance de cette exception historique dans la correspondance graphiephonie. Une dernière étude dans le cadre d'IPFC, celle d'Isely et al. 2018, se base sur le corpus IPFC-alémanique (9 locuteurs de niveau C1). Les auteurs observent notamment un effet de la tâche : tous contextes confondus, le taux d'élision s'élève à $8,63 \%$ en lecture, contre $36,82 \%$ en parole spontanée. Andreassen et Lyche (2018: 19-20) présentent des résultats similaires pour la parole spontanée d'apprenants norvégiens ( 8 de Tromsø de niveau A1 et 8 d'Oslo de niveau B1/B2) : dans le contexte après une seule consonne (VC@C), les débutants laissent tomber $1 \%(3 / 329)$ des schwas dans les monosyllabes et les apprenants avancés $12 \%(84 / 698)$; dans les premières syllabes de polysyllabes, les résultats révèlent des taux d'élision de seulement $2 \%(1 / 41)$ chez les débutants et $14 \%$ $(10 / 70)$ chez les apprenants avancés. Les auteures soulignent l'impact des constructions figées fréquentes: 'Les locutions un petit peu et tout le monde sont

\footnotetext{
${ }^{7} \mathrm{VC} @ \mathrm{C}$ désigne le contexte après une seule consonne à l'intérieur du mot (e.g. chang(e)ment), V\#C@\#C le

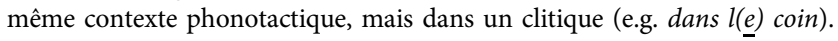


prononcées quasi systématiquement sans schwa et contribuent largement aux pourcentages obtenus à Oslo du fait de leur fréquence dans le corpus' (Andreassen et Lyche, 2018 : 20). De plus, elles soulignent que le schwa tombe particulièrement souvent après les fricatives, notamment dans le pronom clitique très fréquent je dans un syntagme verbal (p. ex. je suis, je sais pas, je trouve). Comme la majorité des apprenants sont traditionnellement exposés à un style très formel et à la forme écrite, la variation du schwa, faisant partie de la compétence sociolinguistique, est peu maîtrisée par des apprenants du français langue étrangère ( $c f$. Dewaele, 2004).

En conclusion, les résultats des études présentées ci-dessus montrent principalement deux tendances : d'une part, le maintien du schwa dans les mots monosyllabiques et en première syllabe de mots polysyllabiques et, d'autre part, l'élision du schwa en syllabe interne et finale de mots polysyllabiques. Étant donné que la recherche sur le schwa en FLE s'est concentrée essentiellement sur les apprenants intermédiaires et avancés (exception faite de Andreassen et Lyche, 2018), souvent étudiants de français, notre projet Pro $^{2} \mathrm{~F}$ a pour objectif de mener des recherches fondamentales sur l'acquisition du schwa chez des apprenants débutants (A1-B1) en milieu scolaire. Les études existantes nous procurent une première impression de ce qui pourra être attendu de notre corpus et nous mènent aux questions de recherche suivantes : 1) De quelle manière la tâche (parole (semi-)spontanée en entretien guidé vs. parole élicitée dans différentes tâches avec et sans présentation de la forme graphique) influence-t-elle la production du schwa des apprenants autrichiens ? 2) Et en particulier : la tâche de répétition en tant que méthode d'élicitation sans présentation de la forme graphique mène-t-elle à des données plus valables et comparables que la tâche de lecture?

\section{METHODE}

Nous avons, sur la base de l'expérience du programme de recherche PFC et de son volet IPFC ( $c f$. section 2.1), mis en place une méthodologie adaptée aux besoins spécifiques du projet Pronunciation in Progress : French Schwa and Liaison (Pro ${ }^{2} \mathrm{~F} ; c f$. https://pro2f.univie.ac.at). Nous présentons ci-dessous les participants de l'enquête (section 3.1), les tâches enregistrées (section 3.2) et la procédure de codage du schwa (section 3.3).

\subsection{Participants de l'enquête}

Le projet de recherche Pro $^{2} \mathrm{~F}$ étudie le français de 145 apprenants autrichiens et plus précisément celui d'élèves ayant entre 12 et 18 ans et étudiant le français comme deuxième langue étrangère, après l'anglais, dans un lycée de Vienne ${ }^{8}$. Les participants de l'enquête se trouvent entre la $1^{\text {ère }}$ et la $6^{\text {ème }}$ année d'apprentissage et devraient se situer - selon les programmes scolaires - entre le niveau débutant (2-6 mois d'apprentissage) et le niveau B1 (intermédiaire) du Cadre Européen Commun de Référence pour les langues (CECR). Le niveau B1 est le

\footnotetext{
${ }^{8} \mathrm{La}$ ou une des L1 des apprenants de notre corpus est toujours l'allemand (en général autrichien). De plus, $29 \%$ de nos élèves sont bilingues avec une deuxième L1 : anglais, arabe, bosniaque, bulgare, chinois, croate, danois, espagnol, grec, hongrois, italien, polonais, portugais, roumain, russe, serbe et tchèque.
} 
Tableau 1. Nombre de participants ${ }^{9}$

\begin{tabular}{|lccc|}
\hline & \multicolumn{3}{c}{ Nombre de participants } \\
\cline { 2 - 4 } Année d'apprentissage & total & f. & $\mathrm{m}$. \\
\hline $1^{\text {ère }}$ & 31 & 18 & 13 \\
\hline $2^{\text {ème }}$ & 21 & 16 & 5 \\
\hline $3^{\text {ème }}$ & 34 & 26 & 8 \\
\hline $4^{\text {ème }}$ & 20 & 17 & 3 \\
\hline $5^{\text {ème }}$ & 20 & 13 & 7 \\
\hline $6^{\text {ème }}$ & 19 & 14 & 5 \\
\hline total & 145 & 104 & 41 \\
\hline & & & \\
\hline
\end{tabular}

niveau cible après six années de français à l'école, mais il ne s'agit que d'un niveau théorique, car les compétences des élèves varient beaucoup, seulement certains élèves atteignent le niveau B1. Le Tableau 1 montre en détail le nombre des participants par année d'apprentissage et sexe. Les enregistrements ont été effectués entre novembre 2017 et avril 2018.

Dans le lycée en question, l'enseignement du français se base sur les manuels scolaires Bien Fait! (1, 2 et 3 ; Luner et al., 2014 ; Luner et Berschotteau 2015, 2017) et Parcours plus (Wlasak-Feik et al., 2016). Ces manuels ne traitent la prononciation que de manière marginale et ne mentionnent le schwa nulle part.

\subsection{Tâches}

Pour chaque participant, nous avons enregistré les tâches suivantes : une lecture de la liste de mots et du texte du programme de recherche PFC (cf. section 2.1) ainsi que d'une nouvelle liste de mots et de deux nouveaux textes (un en français et un autre en allemand) adaptés aux besoins du projet $\operatorname{Pro}^{2} \mathrm{~F}^{10}$. La liste de mots $\operatorname{Pro}^{2} \mathrm{~F}$ comprend la liste de mots IPFC-allemand (cf. Chervinski, 2013) ainsi que des contextes de schwa et de liaison qui sont aussi testés dans une tâche de répétition selon le modèle de lecture d'un présentateur de radio de la région parisienne que nous avons choisi en tant que modèle, ce groupe de locuteurs étant considéré comme représentant le français standard ( $c f$. section 1 et 2.1). Notons qu'il lit la liste de mots Pro $^{2} \mathrm{~F}$ de façon conforme aux règles de prononciation des ouvrages normatifs sans que nous ne le lui ayons donné d'instruction en ce sens ( $c f$. section 1). Le Tableau 2 donne un aperçu sur la prononciation standard et du locuteur modèle des huit cas analysés : notre

\footnotetext{
${ }^{9}$ Les élèves de la $1^{1 \text { ère }}$ année proviennent de trois classes différentes, ceux de la $2^{\text {ème }}$ année de deux classes, à partir de la $3^{\text {ème }}$ année, il n'y a qu'une seule classe.

${ }^{10}$ Nous avons adopté l'ordre suivant : liste de mots $\operatorname{Pro}^{2} \mathrm{~F}$, liste de mots PFC, texte Pro ${ }^{2} \mathrm{~F}$, texte PFC, tâche de répétition, interview guidé.
} 
Tableau 2. Prononciation des mots analysés selon la prononciation standard et du locuteur modèle

\begin{tabular}{lll}
\hline & Standard & Locuteur modèle \\
\hline se_maine & variable & réalisé \\
\hline chęmise & variable & réalisé \\
premier & catégorique & réalisé \\
monsieur & variable & réalisé \\
\hline appartement & catégorique & réalisé \\
\hline gouvernement & catégorique & réalisé \\
tout le temps & élidé & élidé \\
\hline tout le monde & élidé & élidé \\
\hline
\end{tabular}

Tableau 3. Niveaux d'introduction et nombre d'occurrences des mots analysés dans le manuel scolaire des participants Bien Fait!

\begin{tabular}{|c|c|c|c|c|}
\hline \multirow[b]{2}{*}{ Mot } & \multirow{2}{*}{$\begin{array}{c}\text { Première apparition } \\
\begin{array}{c}\text { Bien Fait ! } \\
\text { (volume/unité) }\end{array}\end{array}$} & \multicolumn{3}{|c|}{ Nombre d'occurrences } \\
\hline & & Bien Fait! 1 (A1) & Bien Fait ! 2 (A2) & Bien Fait ! $3(\mathrm{~B} 1+)$ \\
\hline$s(\underline{e})$ maine & $1 / 11,16$ & 13 & 22 & 11 \\
\hline ch(e)mise & $1 / 14$ & 5 & 2 & - \\
\hline$M($ on) sieur & $1 / 2$ & 26 & 14 & 8 \\
\hline premier & $1 / 6$ & 8 & 6 & 8 \\
\hline tout l(e) temps & $1 / 5$ & 5 & 4 & 1 \\
\hline tout l(e) monde & $1 / 5$ & 4 & 8 & 2 \\
\hline appartement & $1 / 1$ & 5 & 10 & 5 \\
\hline gouvernement & $2 / 2$ & - & 1 & 6 \\
\hline
\end{tabular}

présentateur de radio réalise en effet en lecture tous les schwas catégoriques et variables et n'élide que les schwas systématiquement élidés (dont la forme lexicale semble être figée sans schwa ; $c f$. Pustka, 2007).

Le Tableau 3 montre dans quelle leçon les huit mots analysés dans le cadre du présent article sont introduits dans le manuel scolaire utilisé dans le lycée de nos élèves et combien de fois ils y apparaissent. Tous les mots sauf gouvernement sont introduits dans le premier volume du manuel et on peut donc s'attendre à ce qu'ils soient connus dès le niveau A1. On observe cependant de grandes différences concernant le nombre d'occurrences dans le manuel scolaire : Monsieur (26 occ.) et semaine (13 occ.) sont en fait les mots apparaissant le plus 
souvent. Étant donné que ces mots apparaissent en début de groupe dans notre liste de mots, le schwa y est réalisé par notre locuteur modèle. ${ }^{11}$

Quant à la parole spontanée, nous tenons à préciser qu'il s'agit d'un entretien basé sur un guide adapté aux niveaux de compétence des élèves. Les sujets traités dans l'entretien sont, entre autres, la famille, l'école, le temps libre, les vêtements, les fêtes. Tous ces thèmes apparaissent au début du manuel Bien Fait! utilisé pour les élèves de $1^{\text {ère }}$ année.

$\mathrm{Au}$ total, le corpus $\operatorname{Pro}^{2} \mathrm{~F}$ contient 108 heures et 45 minutes de parole et le souscorpus analysé dans le cadre de cet article 87 heures et 10 minutes. Dans le cadre de cette étude 62.535 occurrences potentielles de schwa sont analysées, dont 11.990 se trouvent dans le texte Pro $^{2}$ F, 21.986 dans le texte PFC, 8.418 dans la liste de mots, 4.152 dans la tâche de répétition et 15.989 dans les entretiens guidés.

\subsection{Codage Schwa}

Pour ce qui est de l'analyse du schwa, les enregistrements transcrits sous Praat (cf. Boersma et Weenink, 2018) ont été annotés selon un codage numérique inséré directement après $<\mathrm{e}\rangle$. Ce codage est basé sur celui du programme $\mathrm{PFC}$, mais adapté aux besoins du projet Pro $^{2} \mathrm{~F}$. Chaque code est composé de quatre ou cinq chiffres ( $c f$. Tableau 4). La première position correspond - comme dans le codage PFC - à la présence ou l'absence du schwa. Trois variantes spécifiques aux apprenants autrichiens s'ajoutent ici aux variantes 0 à 2 du codage PFC : $[\mathrm{e}] /[\varepsilon](c f$. section 2.2), d'autres voyelles (p. ex. [0̃], [i], etc. dans Monsieur ; cf. section 4.2) ainsi que la réalisation d'un schwa à la place d'une autre voyelle (p. ex. réce4312mment [веsəmã] ; $c f$. section 2.2). La deuxième position correspond à la position du schwa à l'intérieur du mot et est conforme au codage PFC. La troisième position correspond au contexte gauche. Le codage prévoit, en plus du système de PFC, le codage d'un groupe consonantique simplifié par métathèse (p. ex. Ministre3473 MINISTER [ministes $]^{12}$ ) et celui d'un [1] syllabique (p. ex. article0483 [astikl]). La quatrième position correspond au contexte droit et est à nouveau identique au codage PFC. Nous codons tous les graphèmes $<\mathrm{e}>$ et d'autres graphèmes étant exceptionnellement réalisés par un schwa, mais contrairement aux conventions du programme PFC, nous ne codons pas de consonnes finales (p.ex. avec) car les apprenants germanophones ne réalisent pas de schwas épenthétiques. Si un apprenant réalise un schwa épenthétique, nous le codons exceptionnellement (p.ex. avec1412 ma famille). La position 5 du codage est facultative. Nous codons « R » si l'apprenant répète exactement ce que l'enquêtrice a produit dans l'entretien. " $\mathrm{C}$ » est rajouté dans le cas d'une autocorrection de la part de l'élève.

\footnotetext{
${ }^{11}$ D'une manière générale, schwa a tendance à se maintenir en début de phrase accentuelle. Il s'agit bien évidemment d'un contexte assez artificiel pour un substantif, qui est particulier à la tâche de la liste de mots (p. ex. semaine) - contrairement à la semaine ou une semaine à l'intérieur d'une phrase nominale avec article préposée dans la parole continue : dans la semaine (ou ses chemises dans le texte PFC), le schwa se retrouverait en position après une seule consonne, donc dans un contexte variable; dans une semaine, contexte apparaissant dans le texte Pro $^{2} \mathrm{~F}$, le schwa se trouve après deux consonnes devrait donc y être réalisé (cf. Lyche, $2016: 353$ ).

${ }^{12} \mathrm{La}$ forme double avec tilde $(\sim)$ et un deuxième mot (anglais, allemand) en majuscule suivi d'une transcription en A.P.I. désigne des cas hybrides.
} 
Tableau 4. Codage schwa du projet Pro $^{2} \mathrm{~F}$

\begin{tabular}{|c|c|c|}
\hline Position & Variable & Variantes \\
\hline \multirow[t]{6}{*}{1} & \multirow[t]{6}{*}{ Présence/absence de schwa } & $0=$ pas de schwa \\
\hline & & $1=$ schwa français \\
\hline & & $2=$ cas incertains \\
\hline & & $3=[e] /[\varepsilon]$ \\
\hline & & $4=$ schwa à la place d'une autre voyelle \\
\hline & & $5=$ autre voyelle que $[e] /[\varepsilon]$ ou schwa \\
\hline \multirow[t]{4}{*}{2} & \multirow[t]{4}{*}{ Position à l'intérieur du mot } & $1=$ mot monosyllabique \\
\hline & & $2=$ première syllabe de mot polysyllabique \\
\hline & & $3=$ syllabe interne de mot polysyllabique \\
\hline & & $4=$ syllabe finale de mot polysyllabique \\
\hline \multirow[t]{8}{*}{3} & \multirow[t]{8}{*}{ Contexte gauche } & $1=V_{2}$ \\
\hline & & $2=\mathrm{CC}$ \\
\hline & & $3=$ début de groupe \\
\hline & & $4=$ schwa incertain \\
\hline & & $5=$ groupe consonantique simplifié par élision \\
\hline & & $6=V_{\partial}$ \\
\hline & & $7=$ groupe consonantique simplifié par métathèse \\
\hline & & $8=[l]$ syllabique \\
\hline \multirow[t]{4}{*}{4} & \multirow[t]{4}{*}{ Contexte droit } & $1=$ voyelle \\
\hline & & $2=$ consonne \\
\hline & & $3=$ frontière intonative forte \\
\hline & & $4=$ frontière intonative faible \\
\hline \multirow[t]{2}{*}{5} & \multirow[t]{2}{*}{ Cas spéciaux } & $\mathrm{R}=$ répétition \\
\hline & & $\mathrm{C}=$ autocorrection \\
\hline
\end{tabular}

Les transcriptions et codages ont été effectués dans un premier temps par des étudiantes-assistantes (L1 allemand, niveau $\mathrm{C} 1 / \mathrm{C} 2$ en français ou bilingues $2 \mathrm{~L} 1$ allemand/français) et ensuite revus par les trois auteures de l'article. Pour les cas restant incertains, le codage prévoit le chiffre ' 2 ' en première position.

\section{RESULTATS}

Nos résultats sont exposés dans les Tableau 5 à 12 , dédiés chacun à un mot étudié. Ces tableaux se lisent de la manière suivante : les colonnes indiquent la tâche analysée (répétition d'une liste de mots, lecture de la liste de mots, lecture du texte, parole spontanée en entretien guidé), toutes les tâches n'ayant pas été 
Tableau 5. La réalisation du schwa dans s(e)maine selon la tâche

\begin{tabular}{|c|c|c|c|c|c|c|c|c|c|c|c|c|c|c|c|c|c|c|}
\hline \multirow[b]{2}{*}{ semaine } & & \multicolumn{4}{|c|}{ répétition } & \multicolumn{4}{|c|}{ liste $\operatorname{Pro}^{2} \mathrm{~F}$} & \multicolumn{5}{|c|}{ texte Pro $^{2} \mathrm{~F}$} & \multicolumn{4}{|c|}{ spontané } \\
\hline & & $\varnothing$ & [ə] & ict. & {$[\mathrm{e}] /[\varepsilon]$} & $\varnothing$ & [ə] & ict. & {$[\mathrm{e}] /[\varepsilon]$} & $\varnothing$ & [ə] & ict. & {$[\mathrm{e}] /[\varepsilon]$} & aut. & $\varnothing$ & [ə] & ict. & {$[\mathrm{e}] /[\varepsilon]$} \\
\hline \multirow[t]{6}{*}{$\begin{array}{l}\text { Année } \\
\text { d'apprentissage }\end{array}$} & 1 & - & $\begin{array}{c}97 \% \\
(30 / 31)\end{array}$ & $\begin{array}{c}3 \% \\
(1 / 31)\end{array}$ & - & - & $\begin{array}{c}10 \% \\
(3 / 31)\end{array}$ & $\begin{array}{c}6 \% \\
(2 / 31)\end{array}$ & $\begin{array}{c}84 \% \\
(26 / 31)\end{array}$ & - & $\begin{array}{c}10 \% \\
(3 / 30)\end{array}$ & $\begin{array}{c}3 \% \\
(1 / 30)\end{array}$ & $\begin{array}{c}87 \% \\
(26 / 30)\end{array}$ & - & - & - & $\begin{array}{c}100 \% \\
(1 / 1)\end{array}$ & - \\
\hline & 2 & - & $\begin{array}{c}90 \% \\
(17 / 19)\end{array}$ & - & $\begin{array}{c}10 \% \\
(2 / 19)\end{array}$ & - & $\begin{array}{c}30 \% \\
(7 / 23)\end{array}$ & $\begin{array}{c}35 \% \\
(8 / 23)\end{array}$ & $\begin{array}{c}35 \% \\
(8 / 23)\end{array}$ & - & $\begin{array}{c}40 \% \\
(8 / 20)\end{array}$ & $\begin{array}{c}15 \% \\
(3 / 20)\end{array}$ & $\begin{array}{l}40 \% \\
(8 / 20)\end{array}$ & $\begin{array}{c}5 \% \\
(1 / 20)\end{array}$ & - & $\begin{array}{l}100 \% \\
(2 / 2)\end{array}$ & - & - \\
\hline & 3 & - & $\begin{array}{c}91 \% \\
(31 / 34)\end{array}$ & $\begin{array}{c}9 \% \\
(3 / 34)\end{array}$ & - & - & $\begin{array}{c}42 \% \\
(15 / 36)\end{array}$ & $\begin{array}{c}8 \% \\
(3 / 36)\end{array}$ & $\begin{array}{c}50 \% \\
(18 / 36)\end{array}$ & - & $\begin{array}{c}51 \% \\
(17 / 33)\end{array}$ & $\begin{array}{c}16 \% \\
(5 / 33)\end{array}$ & $\begin{array}{c}33 \% \\
(11 / 33)\end{array}$ & - & - & $\begin{array}{c}71 \% \\
(15 / 21)\end{array}$ & $\begin{array}{c}19 \% \\
(4 / 21)\end{array}$ & $\begin{array}{l}10 \% \\
(2 / 21)\end{array}$ \\
\hline & 4 & - & $\begin{array}{c}79 \% \\
(15 / 19)\end{array}$ & $\begin{array}{c}5 \% \\
(1 / 19)\end{array}$ & $\begin{array}{c}16 \% \\
(3 / 19)\end{array}$ & - & $\begin{array}{c}47 \% \\
(9 / 19)\end{array}$ & $\begin{array}{c}21 \% \\
(4 / 19)\end{array}$ & $\begin{array}{l}32 \% \\
(6 / 19)\end{array}$ & - & $\begin{array}{l}30 \% \\
(6 / 20)\end{array}$ & $\begin{array}{l}10 \% \\
(2 / 20)\end{array}$ & $\begin{array}{c}60 \% \\
(12 / 20)\end{array}$ & - & - & $\begin{array}{l}54 \% \\
(7 / 13)\end{array}$ & $\begin{array}{c}38 \% \\
(5 / 13)\end{array}$ & $\begin{array}{c}8 \% \\
(1 / 13)\end{array}$ \\
\hline & 5 & - & $\begin{array}{c}90 \% \\
(18 / 20)\end{array}$ & - & $\begin{array}{c}10 \% \\
(2 / 20)\end{array}$ & $\begin{array}{l}10 \% \\
(2 / 20)\end{array}$ & $\begin{array}{c}30 \% \\
(6 / 20)\end{array}$ & $\begin{array}{c}50 \% \\
(10 / 20)\end{array}$ & $\begin{array}{l}10 \% \\
(2 / 20)\end{array}$ & - & $\begin{array}{l}35 \% \\
(7 / 20)\end{array}$ & $\begin{array}{c}5 \% \\
(1 / 20)\end{array}$ & $\begin{array}{c}60 \% \\
(12 / 20)\end{array}$ & - & - & $\begin{array}{c}74 \% \\
(22 / 30)\end{array}$ & $\begin{array}{l}26 \% \\
(8 / 30)\end{array}$ & - \\
\hline & 6 & - & $\begin{array}{c}100 \% \\
(17 / 17)\end{array}$ & - & - & - & $\begin{array}{c}72 \% \\
(13 / 18\end{array}$ & $\begin{array}{c}17 \% \\
(3 / 18)\end{array}$ & $\begin{array}{l}11 \% \\
(2 / 18)\end{array}$ & - & $\begin{array}{c}58 \% \\
(11 / 19)\end{array}$ & - & $\begin{array}{c}42 \% \\
(8 / 19)\end{array}$ & - & - & $\begin{array}{c}69 \% \\
(13 / 19)\end{array}$ & $\begin{array}{c}26 \% \\
(5 / 19)\end{array}$ & $\begin{array}{c}5 \% \\
(1 / 19)\end{array}$ \\
\hline tous & & - & $\begin{array}{c}91 \% \\
\left(128 / 140^{13}\right)\end{array}$ & $\begin{array}{c}4 \% \\
(5 / 140)\end{array}$ & $\begin{array}{c}5 \% \\
(7 / 140)\end{array}$ & $\begin{array}{c}1 \% \\
(2 / 147)\end{array}$ & $\begin{array}{c}36 \% \\
(53 / 147)\end{array}$ & $\begin{array}{c}21 \% \\
(30 / 147)\end{array}$ & $\begin{array}{c}42 \% \\
(62 / 147)\end{array}$ & - & $\begin{array}{c}37 \% \\
(52 / 142)\end{array}$ & $\begin{array}{c}8 \% \\
(12 / 142)\end{array}$ & $\begin{array}{c}54 \% \\
(77 / 142)\end{array}$ & $\begin{array}{c}1 \% \\
(1 / 142)\end{array}$ & - & $\begin{array}{c}69 \% \\
(59 / 86)\end{array}$ & $\begin{array}{c}26 \% \\
(23 / 86)\end{array}$ & $\begin{array}{c}5 \% \\
(4 / 86)\end{array}$ \\
\hline
\end{tabular}


testées pour tous les mots. Pour chaque tâche, seules les variantes réalisées sont présentées (élision, schwa, classification incertaine, $[\mathrm{e}] /[\varepsilon]$ et autres variantes). Les résultats pour chaque variante sont présentés en pourcentage et en nombre d'occurrences pour chaque année d'apprentissage et pour l'ensemble du corpus.

L'analyse de notre corpus confirme les grandes tendances phonotactiques observées dans les études existantes ( $c f$. section 2.2 ; Tableau 2 section 3.2) : généralement, les apprenants élident le schwa en syllabe finale (entre $77 \%$ et $94 \%$ selon la tâche) et en syllabe interne après une seule consonne $(86 \%$ d'élisions en lecture et $81 \%$ en parole spontanée). Étant donné que dans ces contextes, les productions en FLE sont très similaires aux productions en français L1 ( $c f$. sections 1 et 2.1), nous les écartons de nos analyses plus détaillées. De plus, nos participants n'élident pratiquement jamais le schwa en première syllabe de mots polysyllabiques et dans les clitiques monosyllabiques, où la réalisation est variable chez les locuteurs L1 du français en parole spontanée et où les apprenants avancés canadiens des études existantes présentent de légers taux d'élision ( $c f$. section 2.2). Ces deux contextes méritent une analyse plus approfondie car l'absence d'élision n'implique pas forcément que la cible visée est un schwa. Les apprenants y réalisent, au contraire, souvent des voyelles erronées en lecture, répétition et parole spontanée, variantes ayant été notées dans différents corpus IPFC-allemand (cf. section 2.2). En lecture, on trouve notamment [e] ou $[\varepsilon]$ (p. ex. dans $54 \%$ des cas dans semaine en lecture du texte; $c f$. section 4.1). Dans le cas de Monsieur, qui contient le digraphe $<$ on $>$, l'on note aussi [o], [0]], [i] et [y], que nous avons regroupés dans une catégorie 'autres' ( $c f$. section 3.3 et 4.2). L'originalité de notre analyse est ainsi qu'elle ne se limite pas à l'opposition entre présence et absence d'une voyelle en contexte schwa, mais qu'elle prend en compte automatiquement sa qualité en cas de présence. Quant aux clitiques, on observe une certaine tendance à l'élision du schwa dans les deux constructions figées tout le monde et tout le temps, qui n’ont, jusqu'ici, guère été analysées en français langue étrangère ( $c f$. section 4.3). Finalement, notre étude confirme que l'élision est fréquente en syllabe interne après deux consonnes dans les cognats anglais appartement et gouvernement, que nous analysons plus en détail dans la section 4.4 .

\subsection{Première syllabe, graphème $<e>$ : $s(\underline{e})$ maine, $\mathrm{ch}(\underline{\mathrm{e}}) \mathrm{mise}$, prẹmier}

Selon l'état actuel de la recherche en français $\mathrm{L} 1$, la réalisation du schwa en première syllabe de mots est catégorique après deux consonnes et après une pause et variable après une seule consonne en parole spontanée ( $c f$. section 2.1). Ainsi, notre locuteurmodèle prononce-t-il premier ainsi que une semaine dans le texte Pro $^{2} \mathrm{~F}$ et semaine dans la liste de mots avec schwa ( $c f$. Tableau 2, section 3.2). C'est également lé cas de ses chemises dans le texte PFC. De plus, la recherche a relevé des différences lexicales notables. Dans le corpus de Hansen 1994, par exemple, le <e > en première syllabe tombe quasiment toujours dans $s(e)$ maine, mais plutôt rarement dans $s(\underline{e})$ crétaire (10\%). Dans notre corpus, des différences lexicales se manifestent plutôt

\footnotetext{
${ }^{13}$ Les nombres totaux varient dû aux erreurs de lecture que nous n'avons pas codés. De plus, le mot Premier apparaît deux fois dans la liste de mots Pro $^{2} \mathrm{~F}$.
} 
rarement ; néanmoins, on observe des différences entre les mots plus ou moins connus par nos élèves, notamment entre semaine et chemise (selon leur appratition dans les manuels; $c f$. Tableau 3) pour ce qui est du taux de réalisation d'un $[e] /[\varepsilon]$ erratique. Concernant la question de la présence ou absence de la voyelle, on observe que les apprenants de notre corpus n'élident jamais le schwa dans $s(\underline{e})$ maine, $\operatorname{ch}(\underline{e})$ mises et premier, et ce, ni en lecture, ni en parole spontanée. Ce comportement correspond dans le cas de la lecture à celui du locuteur modèle et ainsi à la norme de prononciation ( $c f$. Tableau 2) ; en parole spontanée, la réalisation systématique n'est pas erratique, mais témoigne d'un manque de compétence sociolinguistique ( $c f$. Tableaux 5, 6 et 7). Faute d'une observation systématique de l'enseignement en classe, nous ne pouvons pas évaluer les variantes enseignées. Quant à la réalisation de la voyelle, on observe des différences importantes entre les tâches, les années d'apprentissage et les trois mots étudiés. Dans la tâche de répétition, les élèves réalisent le schwa correctement dès la $1^{\text {ère }}$ année à un taux élevé, entre $87 \%$ et $91 \%$ en moyenne. En parole spontanée, ils le réalisent à $69 \%$ dans semaine et à $41 \%$ dans premier (contre 100\% dans les deux seules occurrences de chemise). Dans respectivement $5 \%$ et $35 \%$ des cas, il s'agit cependant d'un $[e] /[\varepsilon]$ et dans $26 \%$ et $21 \%$ des cas d'une voyelle incertaine, c'est-à-dire d'une voyelle se situant entre un schwa et une autre voyelle.

En lecture, en revanche, on observe une progression : dans le cas de semaine, le

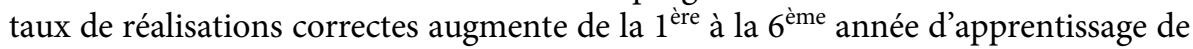
$10 \%$ à $72 \%$ dans la liste de mots et de $10 \%$ à $58 \%$ dans le texte, dans le cas de chemise, il augmente de $12 \%$ à $37 \%$ dans le texte, et dans le cas de premier de $36 \%$ à $58 \%$ dans la liste de mots alors qu'une stagnation autour de $44 \%$ est observable pour ce même mot dans le texte. La plus grande progression, dans le cas de semaine, s'explique probablement par le fait que les élèves se familiarisent davantage avec ce mot : semaine apparaît en effet plus fréquemment dans le manuel scolaire Bien fait ! (46 occurrences) que chemise(s) (7 occurrences; cf. section 3.2), et il est par conséquent aussi utilisé plus fréquemment dans nos enregistrements d'entretiens (86 occurrences de semaine, contrairement à 2 de chemise(s) ; cf. supra). La stagnation observable dans premier dans le texte peut être, en revanche, expliquée par le fait qu'il s'agit du mot composé Premier Ministre auquel correspond le cognat allemand Premierminister, qui en Autriche est prononcé avec la voyelle [e] ; de plus, le cognat allemand Premiere, réalisé avec $[\varepsilon]$, pourrait également expliquer les taux élevés de $[\mathrm{e}] /[\varepsilon]$ en parole spontanée (cf. aussi section 4.4 pour les cognats appartement et gouvernement).

En résumé, la réalisation du schwa dans la première syllabe de mots polysyllabiques est fortement caractérisée par l'influence de la forme graphique en lecture, ce qui explique le taux élevé de la réalisation d'une voyelle erronée [e] ou $[\varepsilon]$ au lieu d'un schwa.

\subsection{Première syllabe, graphème $<$ on $>$ : Monsieur}

D'un point de vue de la structure syllabique, le mot Monsieur présente le même cas que semaine et chemise ( $c f$. section 4.1) : le schwa s'y trouve en première syllabe après une seule consonne. Monsieur constitue pourtant un cas spécifique, car le 
Tableau 6. La réalisation du schwa dans ch(ê)mise selon la tâche

\begin{tabular}{|c|c|c|c|c|c|c|c|c|c|c|c|}
\hline chemise & & \multicolumn{5}{|c|}{ répétition } & \multicolumn{5}{|c|}{ texte PFC } \\
\hline \multirow[t]{6}{*}{ Année d'apprentissage } & 1 & - & $\begin{array}{c}87 \% \\
(27 / 31)\end{array}$ & $\begin{array}{c}7 \% \\
(2 / 31)\end{array}$ & $\begin{array}{c}6 \% \\
(2 / 31)\end{array}$ & - & - & $\begin{array}{c}12 \% \\
(2 / 17)\end{array}$ & $\begin{array}{c}17 \% \\
(3 / 17)\end{array}$ & $\begin{array}{c}59 \% \\
(10 / 17)\end{array}$ & $\begin{array}{c}12 \% \\
(2 / 17)\end{array}$ \\
\hline & 2 & - & $\begin{array}{c}84 \% \\
(16 / 19)\end{array}$ & $\begin{array}{c}16 \% \\
(3 / 19)\end{array}$ & - & - & - & $\begin{array}{c}25 \% \\
(5 / 20)\end{array}$ & $\begin{array}{c}25 \% \\
(5 / 20)\end{array}$ & $\begin{array}{c}50 \% \\
(10 / 20)\end{array}$ & - \\
\hline & 3 & - & $\begin{array}{c}94 \% \\
(32 / 34)\end{array}$ & - & $\begin{array}{c}6 \% \\
(2 / 34)\end{array}$ & - & - & $\begin{array}{c}21 \% \\
(7 / 33)\end{array}$ & $\begin{array}{c}43 \% \\
(14 / 33)\end{array}$ & $\begin{array}{c}36 \% \\
(12 / 33)\end{array}$ & - \\
\hline & 4 & - & $\begin{array}{c}94 \% \\
(17 / 18)\end{array}$ & - & $\begin{array}{c}6 \% \\
(1 / 18)\end{array}$ & - & - & $\begin{array}{c}11 \% \\
(2 / 19)\end{array}$ & $\begin{array}{c}26 \% \\
(5 / 19)\end{array}$ & $\begin{array}{c}58 \% \\
(11 / 19)\end{array}$ & $\begin{array}{c}5 \% \\
(1 / 19)\end{array}$ \\
\hline & 5 & - & $\begin{array}{c}90 \% \\
(18 / 20)\end{array}$ & $\begin{array}{c}5 \% \\
(1 / 20)\end{array}$ & $\begin{array}{c}5 \% \\
(1 / 20)\end{array}$ & - & $\begin{array}{c}5 \% \\
(1 / 21)\end{array}$ & $\begin{array}{c}14 \% \\
(3 / 21)\end{array}$ & $\begin{array}{c}33 \% \\
(7 / 21)\end{array}$ & $\begin{array}{c}48 \% \\
(10 / 21)\end{array}$ & - \\
\hline & 6 & - & $\begin{array}{c}88 \% \\
(15 / 17)\end{array}$ & - & $\begin{array}{c}12 \% \\
(2 / 17)\end{array}$ & - & - & $\begin{array}{c}37 \% \\
(7 / 19)\end{array}$ & $\begin{array}{c}31 \% \\
(6 / 19)\end{array}$ & $\begin{array}{c}32 \% \\
(6 / 19)\end{array}$ & - \\
\hline
\end{tabular}


Tableau 7. La réalisation du schwa dans premier selon la tâche

\begin{tabular}{|c|c|c|c|c|c|c|c|c|c|c|c|c|c|c|c|c|c|}
\hline \multirow[b]{2}{*}{ premier } & & \multicolumn{4}{|c|}{ répétition } & \multicolumn{4}{|c|}{ liste $\operatorname{Pro}^{2} \mathrm{~F}$} & \multicolumn{4}{|c|}{ texte Pro $^{2} \mathrm{~F}$} & \multicolumn{4}{|c|}{ spontané } \\
\hline & & [อ] & ict. & {$[\mathrm{e}] /[\varepsilon]$} & aut. & [ə] & ict. & {$[\mathrm{e}] /[\varepsilon]$} & aut. & [ə] & ict. & {$[e] /[\varepsilon]$} & aut. & [ə] & ict. & {$[\mathrm{e}] /[\varepsilon]$} & aut. \\
\hline \multirow[t]{6}{*}{$\begin{array}{l}\text { Année } \\
\text { d'apprentissage }\end{array}$} & 1 & $\begin{array}{c}82 \% \\
(51 / 62)\end{array}$ & $\begin{array}{c}3 \% \\
(2 / 62)\end{array}$ & $\begin{array}{c}12 \% \\
(7 / 62)\end{array}$ & $\begin{array}{c}3 \% \\
(2 / 62)\end{array}$ & $\begin{array}{c}36 \% \\
(22 / 61)\end{array}$ & $\begin{array}{c}5 \% \\
(3 / 61)\end{array}$ & $\begin{array}{c}57 \% \\
(35 / 61)\end{array}$ & $\begin{array}{c}2 \% \\
(1 / 61)\end{array}$ & $\begin{array}{c}42 \% \\
(13 / 31)\end{array}$ & $\begin{array}{c}3 \% \\
(1 / 31)\end{array}$ & $\begin{array}{c}52 \% \\
(16 / 31)\end{array}$ & $\begin{array}{c}3 \% \\
(1 / 31)\end{array}$ & $\begin{array}{l}33 \% \\
(2 / 6)\end{array}$ & - & $\begin{array}{l}67 \% \\
(4 / 6)\end{array}$ & - \\
\hline & 2 & $\begin{array}{c}89 \% \\
(34 / 38)\end{array}$ & $\begin{array}{c}3 \% \\
(1 / 38)\end{array}$ & $\begin{array}{c}8 \% \\
(3 / 38)\end{array}$ & - & $\begin{array}{c}26 \% \\
(11 / 42)\end{array}$ & - & $\begin{array}{c}69 \% \\
(29 / 42)\end{array}$ & $\begin{array}{c}5 \% \\
(2 / 42)\end{array}$ & $\begin{array}{c}38 \% \\
(8 / 21)\end{array}$ & $\begin{array}{c}9 \% \\
(2 / 21)\end{array}$ & $\begin{array}{c}48 \% \\
(10 / 21)\end{array}$ & $\begin{array}{c}5 \% \\
(1 / 21)\end{array}$ & - & - & - & - \\
\hline & 3 & $\begin{array}{c}88 \% \\
(59 / 67)\end{array}$ & - & $\begin{array}{c}12 \% \\
(8 / 67)\end{array}$ & - & $\begin{array}{c}38 \% \\
(26 / 69)\end{array}$ & $\begin{array}{c}3 \% \\
(2 / 69)\end{array}$ & $\begin{array}{c}58 \% \\
(39 / 69)\end{array}$ & $\begin{array}{c}1 \% \\
(2 / 69)\end{array}$ & $\begin{array}{c}39 \% \\
(13 / 33)\end{array}$ & - & $\begin{array}{c}61 \% \\
(20 / 33)\end{array}$ & - & $\begin{array}{l}50 \% \\
(6 / 12)\end{array}$ & $\begin{array}{c}25 \% \\
(3 / 12)\end{array}$ & $\begin{array}{c}25 \% \\
(3 / 12)\end{array}$ & - \\
\hline & 4 & $\begin{array}{c}84 \% \\
(32 / 38)\end{array}$ & $\begin{array}{c}3 \% \\
(1 / 38)\end{array}$ & $\begin{array}{c}10 \% \\
(4 / 38)\end{array}$ & $\begin{array}{c}3 \% \\
(1 / 38)\end{array}$ & $\begin{array}{c}53 \% \\
(21 / 40)\end{array}$ & $\begin{array}{c}5 \% \\
(2 / 40)\end{array}$ & $\begin{array}{c}40 \% \\
(16 / 40)\end{array}$ & $\begin{array}{c}2 \% \\
(1 / 40)\end{array}$ & $\begin{array}{l}45 \% \\
(9 / 20)\end{array}$ & $\begin{array}{c}5 \% \\
(1 / 20)\end{array}$ & $\begin{array}{c}50 \% \\
(10 / 20)\end{array}$ & - & $\begin{array}{l}43 \% \\
(6 / 14)\end{array}$ & $\begin{array}{l}21 \% \\
(3 / 14)\end{array}$ & $\begin{array}{c}36 \% \\
(5 / 14)\end{array}$ & - \\
\hline & 5 & $\begin{array}{c}90 \% \\
(36 / 40)\end{array}$ & $\begin{array}{c}5 \% \\
(2 / 40)\end{array}$ & $\begin{array}{c}2 \% \\
(1 / 40)\end{array}$ & $\begin{array}{c}3 \% \\
(1 / 40)\end{array}$ & $\begin{array}{c}49 \% \\
(20 / 41)\end{array}$ & $\begin{array}{c}7 \% \\
(3 / 41)\end{array}$ & $\begin{array}{c}41 \% \\
(17 / 41)\end{array}$ & $\begin{array}{c}3 \% \\
(1 / 41)\end{array}$ & $\begin{array}{c}58 \% \\
(11 / 19)\end{array}$ & - & $\begin{array}{c}42 \% \\
(8 / 19)\end{array}$ & - & $\begin{array}{l}33 \% \\
(3 / 9)\end{array}$ & $\begin{array}{l}22 \% \\
(2 / 9)\end{array}$ & $\begin{array}{l}45 \% \\
(4 / 9)\end{array}$ & - \\
\hline & 6 & $\begin{array}{c}94 \% \\
(32 / 34)\end{array}$ & $\begin{array}{c}3 \% \\
(1 / 34)\end{array}$ & $\begin{array}{c}3 \% \\
(1 / 34)\end{array}$ & - & $\begin{array}{c}58 \% \\
(22 / 38)\end{array}$ & $\begin{array}{c}5 \% \\
(2 / 38)\end{array}$ & $\begin{array}{c}32 \% \\
(12 / 38)\end{array}$ & $\begin{array}{l}5 \% \\
(2 / 38)\end{array}$ & $\begin{array}{l}45 \% \\
(9 / 20)\end{array}$ & - & $\begin{array}{c}55 \% \\
(11 / 20)\end{array}$ & - & $\begin{array}{c}41 \% \\
(7 / 17)\end{array}$ & $\begin{array}{c}24 \% \\
(4 / 17)\end{array}$ & $\begin{array}{c}24 \% \\
(4 / 17)\end{array}$ & $\begin{array}{c}12 \% \\
(2 / 17)\end{array}$ \\
\hline tous & & $\begin{array}{c}87 \% \\
(244 / 279)\end{array}$ & $\begin{array}{c}3 \% \\
(7 / 279)\end{array}$ & $\begin{array}{c}9 \% \\
(24 / 279)\end{array}$ & $\begin{array}{c}1 \% \\
(4 / 279)\end{array}$ & $\begin{array}{c}42 \% \\
(122 / 291)\end{array}$ & $\begin{array}{c}4 \% \\
(12 / 291)\end{array}$ & $\begin{array}{c}51 \% \\
(148 / 291)\end{array}$ & $\begin{array}{c}3 \% \\
(9 / 291)\end{array}$ & $\begin{array}{c}44 \% \\
(63 / 144)\end{array}$ & $\begin{array}{c}3 \% \\
(4 / 144)\end{array}$ & $\begin{array}{c}52 \% \\
(75 / 144)\end{array}$ & $\begin{array}{c}1 \% \\
(2 / 144)\end{array}$ & $\begin{array}{c}41 \% \\
(24 / 58)\end{array}$ & $\begin{array}{c}21 \% \\
(12 / 58)\end{array}$ & $\begin{array}{c}35 \% \\
(20 / 58)\end{array}$ & $\begin{array}{c}3 \% \\
(2 / 58)\end{array}$ \\
\hline
\end{tabular}


schwa ne correspond pas à un $<\mathrm{e}>$ graphique comme dans la plupart des cas, mais, pour des raisons étymologiques, à un $<$ on $>$.

Dans notre corpus, les résultats obtenus pour Monsieur divergent ainsi considérablement de ceux pour les autres schwas dans le même contexte phonotactique. Nos apprenants y réalisent en effet très souvent [̃̃], [o], [i] ou [y] au lieu de [ə]. Le taux du code 'autre' (autre voyelle comme p.ex. [õ], [o], [i] ; cf. section 3.3) s'élève à $78 \%$ en moyenne dans la liste de mots, à $69 \%$ dans le texte et à $28 \%$ en répétition, notre corpus ne contenant pas d'occurrences de ce mot en parole spontanée ( $c f$. Tableau 8). Par ailleurs, les taux de cette catégorie 'autre' sont assez stables en lecture, mais diminuent considérablement en répétition, de $37 \%$ en $1^{\text {ère }}$ année à $12 \%$ en $6^{\text {ème }}$ année ; le taux de réalisation $\mathrm{du}$ [ə] augmente parallèlement de $50 \%$ à $76 \%$. Une progression est donc observable. Les réalisations [õ] et [o] s'expliquent facilement par l'application des règles de décodage graphie-phonie régulières de $<$ on $>$ ainsi que par les problèmes bien connus des apprenants germanophones dans la réalisation des voyelles nasalisées du français (cf. Pustka et Meisenburg, 2017 : 133). La réalisation en tant que [i], pour sa part, pourrait être expliquée par le lexème correspondant en anglais Mister.

La prononciation erratique du schwa dans Monsieur s'explique d'une part par l'influence du graphème <on $>$ et d'autre part par le manque d'instruction explicite en salle de classe ( $c f$. section 3.1).

\subsection{Syllabe interne, constructions figées : tout l(e) temps et tout $\mathrm{l}(\mathrm{e})$ monde}

Des recherches sur le français $\mathrm{L} 1$ ont montré que l'élision ou la réalisation du schwa n'est pas uniquement susceptible de se figer dans des lexèmes isolés ( $c f$. section 4.1), mais aussi dans des constructions figées. Ainsi Pustka (2007 : 155, 173-174) montre-t-elle que le schwa se trouvant dans les clitiques après une seule consonne s'élide à $63 \%$ à Paris et à $15 \%$ dans le Sud de la France, alors que dans les constructions figées tout le temps et tout le monde, le taux s'élève à $100 \%$ à Paris et à environ $70 \%$ (tout le temps) et $25 \%$ (tout le monde) dans le Sud. La construction tout le temps semble donc être plus figée que tout le monde.

Dans notre corpus, nous constatons que les apprenants reproduisent les deux constructions avec élision en grande majorité dans la tâche de répétition (94\% et $83 \%$; $c f$. Tableaux 9 et 10 ). En parole spontanée, le taux s'élève à $88 \%$ pour tout le temps, mais à seulement $33 \%$ pour tout le monde (sur seulement 3 occurrences observables). En comparaison, Andreassen et Lyche 2018 avaient relevé 100\% d'élisions dans leur corpus en conversation (cf. section 2.1). En lecture, en revanche, nous avons relevé, dans notre corpus, un taux d'élision d'1\% uniquement pour les deux constructions. Il convient aussi de signaler une régression en répétition au fil des années d'apprentissage : de $100 \%$ à $88 \%$ dans le cas de tout le temps et de $93 \%$ à $67 \%$ dans le cas de tout le monde. La différence entre les deux constructions correspond ici aux différents degrés de figement en L1 (cf. supra), ce qui pourrait être reproduit inconsciemment par les enseignants, qui servent de modèle d'acquisition en salle de classe. Nous supposons donc que les débutants répètent la construction sans l'identifier alors qu'une partie des apprenants 


\begin{tabular}{|c|c|c|c|c|c|c|c|c|c|c|c|c|c|c|c|c|}
\hline \multirow[b]{2}{*}{ Monsieur } & & \multicolumn{5}{|c|}{ répétition } & \multicolumn{5}{|c|}{ liste $\operatorname{Pro}^{2} \mathrm{~F}$} & \multicolumn{5}{|c|}{ texte Pro ${ }^{2} \mathrm{~F}$} \\
\hline & & $\varnothing$ & [ə] & ict. & {$[\mathrm{e}] /[\varepsilon]$} & aut. & $\varnothing$ & [ə] & ict. & {$[\mathrm{e}] /[\varepsilon]$} & aut. & $\varnothing$ & [ə] & ict. & {$[\mathrm{e}] /[\varepsilon]$} & aut. \\
\hline \multirow[t]{6}{*}{ Année d'apprentissage } & 1 & - & $\begin{array}{c}50 \% \\
(15 / 30)\end{array}$ & $\begin{array}{c}3 \% \\
(1 / 30)\end{array}$ & $\begin{array}{c}10 \% \\
(3 / 30)\end{array}$ & $\begin{array}{c}37 \% \\
(11 / 30)\end{array}$ & - & $\begin{array}{c}17 \% \\
(5 / 33)\end{array}$ & $\begin{array}{c}3 \% \\
(1 / 33)\end{array}$ & $\begin{array}{c}9 \% \\
(3 / 33)\end{array}$ & $\begin{array}{c}71 \% \\
(24 / 33)\end{array}$ & $\begin{array}{c}7 \% \\
(2 / 31)\end{array}$ & $\begin{array}{c}16 \% \\
(5 / 31)\end{array}$ & $\begin{array}{c}3 \% \\
(1 / 31)\end{array}$ & $\begin{array}{c}6 \% \\
(2 / 31)\end{array}$ & $\begin{array}{c}68 \% \\
(21 / 31)\end{array}$ \\
\hline & 2 & - & $50 \%(9 / 18)$ & $\begin{array}{c}6 \% \\
(1 / 18)\end{array}$ & $\begin{array}{c}11 \% \\
(2 / 18)\end{array}$ & $\begin{array}{c}33 \% \\
(6 / 18)\end{array}$ & - & $\begin{array}{c}10 \% \\
(2 / 20)\end{array}$ & $\begin{array}{c}5 \% \\
(1 / 20)\end{array}$ & $\begin{array}{l}10 \% \\
(2 / 20)\end{array}$ & $\begin{array}{c}75 \% \\
(15 / 20)\end{array}$ & $\begin{array}{c}5 \% \\
(1 / 21)\end{array}$ & $\begin{array}{c}38 \% \\
(8 / 21)\end{array}$ & - & - & $\begin{array}{c}57 \% \\
12 / 21)\end{array}$ \\
\hline & 3 & - & $\begin{array}{c}44 \% \\
(15 / 34)\end{array}$ & $\begin{array}{c}3 \% \\
(1 / 34)\end{array}$ & $\begin{array}{c}18 \% \\
(6 / 34)\end{array}$ & $\begin{array}{c}35 \% \\
(12 / 34)\end{array}$ & - & $\begin{array}{c}12 \% \\
(4 / 34)\end{array}$ & $\begin{array}{c}3 \% \\
(1 / 34)\end{array}$ & $\begin{array}{c}3 \% \\
(1 / 34)\end{array}$ & $\begin{array}{c}82 \% \\
(28 / 34)\end{array}$ & - & $\begin{array}{c}18 \% \\
(6 / 33)\end{array}$ & - & $\begin{array}{c}3 \% \\
(1 / 33)\end{array}$ & $\begin{array}{c}79 \% \\
(26 / 33)\end{array}$ \\
\hline & 4 & - & $\begin{array}{c}32 \% \\
(6 / 19)\end{array}$ & $\begin{array}{c}5 \% \\
(1 / 19)\end{array}$ & $\begin{array}{c}42 \% \\
(8 / 19)\end{array}$ & $\begin{array}{c}21 \% \\
(4 / 19)\end{array}$ & - & $\begin{array}{c}11 \% \\
(2 / 19)\end{array}$ & $\begin{array}{c}5 \% \\
(1 / 19)\end{array}$ & $\begin{array}{c}5 \% \\
(1 / 19)\end{array}$ & $\begin{array}{c}79 \% \\
(15 / 19)\end{array}$ & - & $\begin{array}{c}35 \% \\
(7 / 20)\end{array}$ & - & - & $\begin{array}{c}65 \% \\
(13 / 20)\end{array}$ \\
\hline & 5 & - & $\begin{array}{c}71 \% \\
(15 / 21)\end{array}$ & - & $\begin{array}{c}10 \% \\
(2 / 21)\end{array}$ & $\begin{array}{c}19 \% \\
(4 / 21)\end{array}$ & - & $\begin{array}{c}18 \% \\
(4 / 22)\end{array}$ & $\begin{array}{c}5 \% \\
(1 / 22)\end{array}$ & $\begin{array}{c}5 \% \\
(1 / 22)\end{array}$ & $\begin{array}{c}72 \% \\
(16 / 22)\end{array}$ & - & $\begin{array}{c}30 \% \\
(6 / 20)\end{array}$ & - & - & $\begin{array}{c}70 \% \\
(14 / 20)\end{array}$ \\
\hline & 6 & - & $\begin{array}{c}76 \% \\
(13 / 17)\end{array}$ & - & $\begin{array}{c}12 \% \\
(2 / 17)\end{array}$ & $\begin{array}{c}12 \% \\
(2 / 17)\end{array}$ & - & $\begin{array}{c}16 \% \\
(3 / 19)\end{array}$ & - & - & $\begin{array}{c}84 \% \\
(16 / 19)\end{array}$ & - & $\begin{array}{c}32 \% \\
(6 / 19)\end{array}$ & $\begin{array}{c}5 \% \\
(1 / 19)\end{array}$ & - & $\begin{array}{c}63 \% \\
(12 / 19)\end{array}$ \\
\hline \multicolumn{2}{|l|}{ tous } & - & $\begin{array}{c}53 \% \\
(73 / 139)\end{array}$ & $\begin{array}{c}3 \% \\
(4 / 139)\end{array}$ & $\begin{array}{c}16 \% \\
(23 / 139)\end{array}$ & $\begin{array}{c}28 \% \\
(39 / 139)\end{array}$ & - & $\begin{array}{c}14 \% \\
(20 / 147)\end{array}$ & $\begin{array}{c}3 \% \\
(5 / 147)\end{array}$ & $\begin{array}{c}5 \% \\
(8 / 147)\end{array}$ & $\begin{array}{c}78 \% \\
(114 / 147)\end{array}$ & $\begin{array}{c}2 \% \\
(3 / 144)\end{array}$ & $\begin{array}{c}26 \% \\
(38 / 144)\end{array}$ & $\begin{array}{c}1 \% \\
(2 / 144)\end{array}$ & $\begin{array}{c}2 \% \\
(3 / 144)\end{array}$ & $\begin{array}{c}69 \% \\
(98 / 144)\end{array}$ \\
\hline
\end{tabular}


Tableau 9. La réalisation du schwa dans tout le temps selon la tâche

\begin{tabular}{|c|c|c|c|c|c|c|c|c|c|c|c|c|c|}
\hline \multirow[b]{2}{*}{ tout le temps } & & \multicolumn{4}{|c|}{ répétition } & \multicolumn{4}{|c|}{ liste $\operatorname{Pro}^{2} \mathrm{~F}$} & \multicolumn{4}{|c|}{ spontané } \\
\hline & & $\varnothing$ & [ə] & ict. & {$[\mathrm{e}] /[\varepsilon]$} & $\varnothing$ & [ə] & ict. & {$[\mathrm{e}] /[\varepsilon]$} & $\varnothing$ & [ə] & ict. & {$[\mathrm{e}] /[\varepsilon]$} \\
\hline \multirow[t]{6}{*}{$\begin{array}{l}\text { Année } \\
\text { d'apprentissage }\end{array}$} & 1 & $\begin{array}{c}100 \% \\
(31 / 31)\end{array}$ & - & - & - & $\begin{array}{c}3 \% \\
(1 / 31)\end{array}$ & $\begin{array}{c}58 \% \\
(18 / 31)\end{array}$ & - & $\begin{array}{c}39 \% \\
(12 / 31)\end{array}$ & - & - & - & - \\
\hline & 2 & $\begin{array}{c}94 \% \\
(17 / 18)\end{array}$ & $\begin{array}{c}6 \% \\
(1 / 18)\end{array}$ & - & - & - & $\begin{array}{c}71 \% \\
(15 / 21)\end{array}$ & $\begin{array}{c}5 \% \\
(1 / 21)\end{array}$ & $\begin{array}{c}24 \% \\
(5 / 21)\end{array}$ & - & - & - & - \\
\hline & 3 & $\begin{array}{c}91 \% \\
(31 / 34)\end{array}$ & $\begin{array}{c}9 \% \\
(3 / 34)\end{array}$ & - & - & - & $\begin{array}{c}79 \% \\
(27 / 34)\end{array}$ & $\begin{array}{c}6 \% \\
(2 / 34)\end{array}$ & $\begin{array}{c}15 \% \\
(5 / 34)\end{array}$ & $\begin{array}{c}100 \% \\
(7 / 7)\end{array}$ & - & - & - \\
\hline & 4 & $\begin{array}{c}95 \% \\
(18 / 19)\end{array}$ & $\begin{array}{c}5 \% \\
(1 / 19)\end{array}$ & - & - & - & $\begin{array}{c}75 \% \\
(15 / 20)\end{array}$ & - & $\begin{array}{c}25 \% \\
(5 / 20)\end{array}$ & $\begin{array}{l}80 \% \\
(4 / 5)\end{array}$ & - & $\begin{array}{l}20 \% \\
(1 / 5)\end{array}$ & \\
\hline & 5 & $\begin{array}{c}90 \% \\
(18 / 20)\end{array}$ & $\begin{array}{c}10 \% \\
(2 / 20)\end{array}$ & - & - & $\begin{array}{c}5 \% \\
(1 / 20)\end{array}$ & $\begin{array}{c}80 \% \\
(16 / 20)\end{array}$ & $\begin{array}{c}10 \% \\
(2 / 20)\end{array}$ & $\begin{array}{c}5 \% \\
(1 / 20)\end{array}$ & $\begin{array}{c}100 \% \\
(6 / 6)\end{array}$ & - & - & \\
\hline & 6 & $\begin{array}{c}88 \% \\
(15 / 17)\end{array}$ & $\begin{array}{c}6 \% \\
(1 / 17)\end{array}$ & $\begin{array}{c}6 \% \\
(1 / 17)\end{array}$ & - & $\begin{array}{c}6 \% \\
(1 / 18)\end{array}$ & $\begin{array}{c}78 \% \\
(14 / 18)\end{array}$ & $\begin{array}{c}16 \% \\
(3 / 18)\end{array}$ & - & $\begin{array}{l}75 \% \\
(6 / 8)\end{array}$ & - & $\begin{array}{l}13 \% \\
/ 1 / 8)\end{array}$ & $\begin{array}{l}12 \% \\
(1 / 8)\end{array}$ \\
\hline tous & & $\begin{array}{c}94 \% \\
(130 / 139)\end{array}$ & $\begin{array}{c}6 \% \\
(8 / 139)\end{array}$ & $\begin{array}{c}1 \% \\
(1 / 139)\end{array}$ & - & $\begin{array}{c}2 \% \\
(3 / 144)\end{array}$ & $\begin{array}{c}73 \% \\
(105 / 144)\end{array}$ & $\begin{array}{c}3 \% \\
(5 / 144)\end{array}$ & $\begin{array}{c}22 \% \\
(31 / 144)\end{array}$ & $\begin{array}{c}88 \% \\
(23 / 26)\end{array}$ & - & $\begin{array}{c}8 \% \\
(2 / 26)\end{array}$ & $\begin{array}{c}4 \% \\
(1 / 26)\end{array}$ \\
\hline
\end{tabular}




\begin{tabular}{|c|c|c|c|c|c|c|c|c|c|c|c|c|c|}
\hline \multirow[b]{2}{*}{ tout le monde } & & \multicolumn{4}{|c|}{ répétition } & \multicolumn{4}{|c|}{ texte $\operatorname{Pro}^{2} \mathrm{~F}$} & \multicolumn{4}{|c|}{ spontané } \\
\hline & & $\varnothing$ & [ə] & ict. & {$[\mathrm{e}] /[\varepsilon]$} & $\varnothing$ & [ə] & ict. & {$[\mathrm{e}] /[\varepsilon]$} & $\varnothing$ & [ə] & ict. & {$[\mathrm{e}] /[\varepsilon]$} \\
\hline \multirow[t]{6}{*}{ Année d'apprentissage } & 1 & $\begin{array}{c}93 \% \\
(28 / 30)\end{array}$ & $\begin{array}{c}3 \% \\
(1 / 30)\end{array}$ & - & $\begin{array}{c}4 \% \\
(1 / 30)\end{array}$ & - & $\begin{array}{c}23 \% \\
(7 / 31)\end{array}$ & - & $\begin{array}{c}77 \% \\
(14 / 31)\end{array}$ & - & - & - & - \\
\hline & 2 & $\begin{array}{c}84 \% \\
(16 / 19)\end{array}$ & $\begin{array}{c}11 \% \\
(2 / 19)\end{array}$ & $\begin{array}{c}5 \% \\
(1 / 19)\end{array}$ & - & - & $\begin{array}{c}70 \% \\
(14 / 20)\end{array}$ & $\begin{array}{c}10 \% \\
(2 / 20)\end{array}$ & $\begin{array}{c}20 \% \\
(4 / 20)\end{array}$ & - & - & - & - \\
\hline & 3 & $\begin{array}{c}73 \% \\
(22 / 30)\end{array}$ & $\begin{array}{c}23 \% \\
(7 / 30)\end{array}$ & - & $\begin{array}{c}4 \% \\
(1 / 30)\end{array}$ & - & $\begin{array}{c}75 \% \\
(24 / 32)\end{array}$ & $\begin{array}{c}6 \% \\
(2 / 32)\end{array}$ & $\begin{array}{c}19 \% \\
(6 / 32)\end{array}$ & - & $\begin{array}{l}100 \% \\
(1 / 1)\end{array}$ & - & - \\
\hline & 4 & $\begin{array}{c}85 \% \\
(11 / 13)\end{array}$ & $\begin{array}{c}15 \% \\
(2 / 13)\end{array}$ & - & - & - & $\begin{array}{c}80 \% \\
(16 / 20)\end{array}$ & $\begin{array}{l}10 \% \\
(2 / 20)\end{array}$ & $\begin{array}{c}10 \% \\
(2 / 20)\end{array}$ & - & - & - & - \\
\hline & 5 & $\begin{array}{c}88 \% \\
(14 / 16)\end{array}$ & $\begin{array}{c}6 \% \\
(1 / 16)\end{array}$ & $\begin{array}{c}6 \% \\
(1 / 16)\end{array}$ & - & $\begin{array}{c}5 \% \\
(1 / 18)\end{array}$ & $\begin{array}{c}78 \% \\
(14 / 18)\end{array}$ & $\begin{array}{c}5 \% \\
(1 / 18)\end{array}$ & $\begin{array}{c}12 \% \\
(2 / 18)\end{array}$ & $\begin{array}{c}100 \% \\
(1 / 1)\end{array}$ & - & - & - \\
\hline & 6 & $\begin{array}{c}67 \% \\
(8 / 12)\end{array}$ & $\begin{array}{c}17 \% \\
(2 / 12)\end{array}$ & $\begin{array}{c}16 \% \\
(2 / 12)\end{array}$ & - & $\begin{array}{c}5 \% \\
(1 / 18)\end{array}$ & $\begin{array}{c}83 \% \\
(15 / 18)\end{array}$ & - & $\begin{array}{c}12 \% \\
(2 / 18)\end{array}$ & - & - & $\begin{array}{c}100 \% \\
(1 / 1)\end{array}$ & - \\
\hline tous & & $\begin{array}{c}83 \% \\
(99 / 120)\end{array}$ & $\begin{array}{c}13 \% \\
(15 / 120)\end{array}$ & $\begin{array}{c}3 \% \\
(4 / 120)\end{array}$ & $\begin{array}{c}1 \% \\
(2 / 120)\end{array}$ & $\begin{array}{c}1 \% \\
(2 / 139)\end{array}$ & $\begin{array}{c}65 \% \\
(90 / 139)\end{array}$ & $\begin{array}{c}5 \% \\
(7 / 139)\end{array}$ & $\begin{array}{c}29 \% \\
(40 / 139)\end{array}$ & $\begin{array}{l}33 \% \\
(1 / 3)\end{array}$ & $\begin{array}{l}33 \% \\
(1 / 3)\end{array}$ & $\begin{array}{l}34 \% \\
(1 / 3)\end{array}$ & - \\
\hline
\end{tabular}


intermédiaires reconnaissent les mots entendus et les associent aux représentations cognitives qu'ils en ont développées à partir de la forme graphique.

\subsection{Syllabe interne, cognats : appartement et gouvernement}

Les études sur le schwa en FLE ont montré que les cognats présentent un défi particulier pour les apprenants ( $c f$. section 2.2). Alors que les locuteurs du français $\mathrm{L} 1$ réalisent le schwa en position interne après deux consonnes presque systématiquement, nous savons que ceci n'est pas le cas chez les apprenants anglophones, notamment dans les mots appartement et gouvernement, étant donnée l'influence des cognats anglais apartment et government. Chez les apprenants autrichiens, il faudrait ajouter le cognat allemand Appartment, prononcé à l'anglaise sans schwa, ce qui se reflète aussi dans la graphie, ainsi que l'influence de l'anglais en tant que première langue étrangère. Pour le cas spécifique de gouvernement se trouvant dans le texte PFC, les analyses du corpus PFC montrent que chez quelques locuteurs L1 du français, le schwa n'est pas non plus réalisé (cf. section 2.2).

Les résultats obtenus à partir de notre corpus confirment le comportement particulier du schwa dans les cognats chez les apprenants (cf. Tableaux 11 et 12). Contrairement aux lexèmes étudiés dans les sections précédentes, les réalisations en parole spontanée (si rares qu'elles soient) divergent fortement de celles observables en répétition : en parole spontanée, $100 \%$ des schwas sont élidés dans appartement (14/14) et gouvernement (2/2). Dans la tâche de répétition, en revanche, $78 \%$ des apprenants réalisent le schwa correctement dans les deux mots. En lecture, les pourcentages se situent entre ces deux extrêmes : dans appartement, $77 \%$ des schwas sont élidés dans la liste de mots et $81 \%$ dans le texte, dans gouvernement 59\% des schwas sont élidés dans le texte. L'élision catégorique en parole spontanée s'explique par l'influence des cognats et la différence entre les deux mots en lecture éventuellement par la seule existence du cognat Appartment en allemand.

Les mots premier, appartement et gouvernement montrent ainsi que l'influence de la L1 (allemand) ainsi que de la première langue étrangère (anglais) ne se limitent pas aux niveaux des sons et phonèmes, mais concernent aussi des représentations de lexèmes entiers.

\section{CONCLUSION}

L'objectif de cet article était méthodologique. Il visait à montrer l'impact de la tâche choisie sur la réalisation du schwa français par des élèves autrichiens. Nous avons comparé à cette fin en tant que tâches d'élicitation la lecture de listes de mots et de textes et la répétition à une tâche (semi-)spontanée, l'entretien guidé. Cette comparaison nous permet d'évaluer la validité et la comparabilité des données et de perfectionner la méthodologie de la phonologie de corpus.

L'analyse de notre corpus montre encore une fois à quel point il est important de combiner différentes méthodes de recherche pour un même phénomène (cf. section 2.1). Même un large corpus de plus de 35 heures de parole spontanée, qui constitue la méthode la plus valable pour l'analyse du système linguistique, ne présente que peu ou pas d'occurrences pour une grande partie 
Tableau 11. La réalisation du schwa dans appartement selon la tâche

\begin{tabular}{|c|c|c|c|c|c|c|c|c|c|c|c|c|c|c|c|c|c|}
\hline \multirow[b]{2}{*}{ appartement } & & \multicolumn{4}{|c|}{ répétition } & \multicolumn{4}{|c|}{ liste Pro $^{2} \mathrm{~F}$} & \multicolumn{4}{|c|}{ texte $\operatorname{Pro}^{2} \mathrm{~F}$} & \multicolumn{4}{|c|}{ spontané } \\
\hline & & $\varnothing$ & [ə] & ict. & {$[\mathrm{e}] /[\varepsilon]$} & $\varnothing$ & [ə] & ict. & {$[\mathrm{e}] /[\varepsilon]$} & $\varnothing$ & [ə] & ict. & {$[\mathrm{e}] /[\varepsilon]$} & $\varnothing$ & [ə] & ict. & {$[\mathrm{e}] /[\varepsilon]$} \\
\hline \multirow[t]{6}{*}{ Année d'apprentissage } & 1 & $\begin{array}{c}10 \% \\
(3 / 31)\end{array}$ & $\begin{array}{c}71 \% \\
(22 / 31)\end{array}$ & $\begin{array}{c}16 \% \\
(5 / 31)\end{array}$ & $\begin{array}{c}3 \% \\
(1 / 31)\end{array}$ & $\begin{array}{c}65 \% \\
(20 / 31)\end{array}$ & $\begin{array}{c}22 \% \\
(7 / 31)\end{array}$ & $\begin{array}{c}3 \% \\
(1 / 31)\end{array}$ & $\begin{array}{c}10 \% \\
(3 / 31)\end{array}$ & $\begin{array}{c}70 \% \\
(21 / 30)\end{array}$ & $\begin{array}{c}17 \% \\
(5 / 30)\end{array}$ & - & $\begin{array}{c}13 \% \\
(4 / 30)\end{array}$ & - & - & - & - \\
\hline & 2 & $\begin{array}{c}15 \% \\
(3 / 20)\end{array}$ & $\begin{array}{c}75 \% \\
(15 / 20)\end{array}$ & $\begin{array}{c}5 \% \\
(1 / 20)\end{array}$ & $\begin{array}{c}5 \% \\
(1 / 20)\end{array}$ & $\begin{array}{c}81 \% \\
(17 / 21)\end{array}$ & $\begin{array}{c}14 \% \\
(3 / 21)\end{array}$ & - & $\begin{array}{c}5 \% \\
(1 / 21)\end{array}$ & $\begin{array}{c}76 \% \\
(16 / 21)\end{array}$ & $\begin{array}{c}14 \% \\
(3 / 21)\end{array}$ & $\begin{array}{c}10 \% \\
(2 / 21)\end{array}$ & - & $\begin{array}{c}100 \% \\
(1 / 1)\end{array}$ & - & - & - \\
\hline & 3 & $\begin{array}{c}12 \% \\
(4 / 34)\end{array}$ & $\begin{array}{c}82 \% \\
(28 / 34)\end{array}$ & - & $\begin{array}{c}6 \% \\
(2 / 34)\end{array}$ & $\begin{array}{c}83 \% \\
(19 / 35)\end{array}$ & $\begin{array}{c}11 \% \\
(4 / 35)\end{array}$ & $\begin{array}{c}3 \% \\
(1 / 35)\end{array}$ & $\begin{array}{c}3 \% \\
(1 / 35)\end{array}$ & $\begin{array}{c}94 \% \\
(31 / 33)\end{array}$ & $\begin{array}{c}6 \% \\
(2 / 33)\end{array}$ & - & - & $\begin{array}{c}100 \% \\
(5 / 5)\end{array}$ & - & - & - \\
\hline & 4 & $\begin{array}{c}5 \% \\
(1 / 19)\end{array}$ & $\begin{array}{c}84 \% \\
(16 / 19)\end{array}$ & $\begin{array}{c}11 \% \\
(2 / 19)\end{array}$ & - & $\begin{array}{c}90 \% \\
(18 / 20)\end{array}$ & $\begin{array}{c}10 \% \\
(2 / 20)\end{array}$ & - & - & $\begin{array}{c}95 \% \\
(19 / 20)\end{array}$ & $\begin{array}{c}5 \% \\
(1 / 20)\end{array}$ & - & - & $\begin{array}{c}100 \% \\
(2 / 2)\end{array}$ & - & - & - \\
\hline & 5 & $\begin{array}{c}5 \% \\
(1 / 20)\end{array}$ & $\begin{array}{c}75 \% \\
(15 / 20)\end{array}$ & $\begin{array}{c}5 \% \\
(1 / 20)\end{array}$ & $\begin{array}{c}15 \% \\
(3 / 20)\end{array}$ & $\begin{array}{c}76 \% \\
(16 / 21)\end{array}$ & $\begin{array}{c}19 \% \\
(4 / 21)\end{array}$ & - & $\begin{array}{c}5 \% \\
(1 / 21)\end{array}$ & $\begin{array}{c}75 \% \\
(15 / 20)\end{array}$ & $\begin{array}{c}15 \% \\
(3 / 20)\end{array}$ & $\begin{array}{c}5 \% \\
(1 / 20)\end{array}$ & $\begin{array}{c}5 \% \\
(1 / 20)\end{array}$ & $\begin{array}{c}100 \% \\
(4 / 4)\end{array}$ & - & - & - \\
\hline & 6 & $\begin{array}{c}6 \% \\
(1 / 17)\end{array}$ & $\begin{array}{c}82 \% \\
(14 / 17)\end{array}$ & $\begin{array}{c}6 \% \\
(1 / 17)\end{array}$ & $\begin{array}{c}6 \% \\
(1 / 17)\end{array}$ & $67 \%(12 / 18)$ & $\begin{array}{c}28 \% \\
(5 / 18)\end{array}$ & $\begin{array}{c}5 \% \\
(1 / 18)\end{array}$ & - & $\begin{array}{c}74 \% \\
(14 / 19)\end{array}$ & $\begin{array}{c}16 \% \\
(3 / 19)\end{array}$ & $\begin{array}{c}10 \% \\
(2 / 19)\end{array}$ & - & $\begin{array}{c}100 \% \\
(2 / 2)\end{array}$ & - & - & - \\
\hline tous & & $\begin{array}{c}9 \% \\
(13 / 141)\end{array}$ & $\begin{array}{c}78 \% \\
(110 / 141)\end{array}$ & $\begin{array}{c}7 \% \\
(10 / 141)\end{array}$ & $\begin{array}{c}6 \% \\
(8 / 141)\end{array}$ & $\begin{array}{c}77 \% \\
(112 / 146)\end{array}$ & $\begin{array}{c}17 \% \\
(25 / 146)\end{array}$ & $\begin{array}{c}2 \% \\
(3 / 146)\end{array}$ & $\begin{array}{c}4 \% \\
(6 / 146)\end{array}$ & $\begin{array}{c}81 \% \\
(116 / 143)\end{array}$ & $\begin{array}{c}12 \% \\
(17 / 143)\end{array}$ & $\begin{array}{c}3 \% \\
(5 / 143)\end{array}$ & $\begin{array}{c}4 \% \\
(5 / 143)\end{array}$ & $\begin{array}{c}100 \% \\
(14 / 14)\end{array}$ & - & - & - \\
\hline
\end{tabular}


Tableau 12. La réalisation du schwa dans gouvernement selon la tâche

\begin{tabular}{|c|c|c|c|c|c|c|c|c|c|c|c|c|c|}
\hline \multirow[b]{2}{*}{ gouvernement } & & \multicolumn{4}{|c|}{ répétition } & \multicolumn{4}{|c|}{ texte PFC } & \multicolumn{4}{|c|}{ spontané } \\
\hline & & $\varnothing$ & [ə] & ict. & {$[\mathrm{e}] /[\varepsilon]$} & $\varnothing$ & [ə] & ict. & {$[\mathrm{e}] /[\varepsilon]$} & $\varnothing$ & [ə] & ict. & {$[\mathrm{e}] /[\varepsilon]$} \\
\hline \multirow[t]{6}{*}{ Année d'apprentissage } & 1 & $\begin{array}{c}3 \% \\
(1 / 29)\end{array}$ & $\begin{array}{c}66 \% \\
(19 / 29)\end{array}$ & $\begin{array}{c}14 \% \\
(4 / 29)\end{array}$ & $\begin{array}{c}17 \% \\
(5 / 29)\end{array}$ & $\begin{array}{c}62 \% \\
(8 / 13)\end{array}$ & $\begin{array}{c}8 \% \\
(1 / 13)\end{array}$ & - & $\begin{array}{c}31 \% \\
(4 / 13)\end{array}$ & - & - & - & - \\
\hline & 2 & $\begin{array}{c}16 \% \\
(3 / 19)\end{array}$ & $\begin{array}{c}79 \% \\
(15 / 19)\end{array}$ & - & $\begin{array}{c}5 \% \\
(1 / 19)\end{array}$ & $\begin{array}{c}45 \% \\
(5 / 11)\end{array}$ & $\begin{array}{c}45 \% \\
(5 / 11)\end{array}$ & - & $\begin{array}{c}10 \% \\
(1 / 11)\end{array}$ & - & - & - & - \\
\hline & 3 & $\begin{array}{c}6 \% \\
(2 / 34)\end{array}$ & $\begin{array}{c}76 \% \\
(26 / 34)\end{array}$ & $\begin{array}{c}6 \% \\
(2 / 34)\end{array}$ & $\begin{array}{c}12 \% \\
(4 / 34)\end{array}$ & $\begin{array}{c}50 \% \\
(5 / 10)\end{array}$ & $\begin{array}{c}20 \% \\
(2 / 10)\end{array}$ & $\begin{array}{c}30 \% \\
(3 / 10)\end{array}$ & - & $\begin{array}{c}100 \% \\
(1 / 1)\end{array}$ & - & - & - \\
\hline & 4 & $\begin{array}{c}5 \% \\
(1 / 19)\end{array}$ & $\begin{array}{c}84 \% \\
(16 / 19)\end{array}$ & - & $\begin{array}{c}11 \% \\
(2 / 19)\end{array}$ & $\begin{array}{l}89 \% \\
(8 / 9)\end{array}$ & $\begin{array}{l}11 \% \\
(1 / 9)\end{array}$ & - & - & - & - & - & - \\
\hline & 5 & - & $\begin{array}{c}80 \% \\
(16 / 20)\end{array}$ & $\begin{array}{c}10 \% \\
(2 / 20)\end{array}$ & $\begin{array}{c}10 \% \\
(2 / 20)\end{array}$ & $\begin{array}{c}53 \% \\
(8 / 15)\end{array}$ & $\begin{array}{c}47 \% \\
(7 / 15)\end{array}$ & - & - & - & - & - & - \\
\hline & 6 & $\begin{array}{c}6 \% \\
(1 / 17)\end{array}$ & $\begin{array}{c}76 \% \\
(13 / 17)\end{array}$ & $\begin{array}{c}12 \% \\
(2 / 17)\end{array}$ & $\begin{array}{c}6 \% \\
(1 / 17)\end{array}$ & $\begin{array}{l}58 \% \\
7 / 12)\end{array}$ & $\begin{array}{c}25 \% \\
(3 / 12)\end{array}$ & $\begin{array}{c}17 \% \\
(2 / 12)\end{array}$ & - & $\begin{array}{l}100 \% \\
(1 / 1)\end{array}$ & - & - & - \\
\hline tous & & $\begin{array}{c}6 \% \\
(8 / 139)\end{array}$ & $\begin{array}{c}78 \% \\
(105 / 135)\end{array}$ & $\begin{array}{c}7 \% \\
(10 / 139)\end{array}$ & $\begin{array}{c}11 \% \\
(15 / 139)\end{array}$ & $\begin{array}{c}59 \% \\
(41 / 70)\end{array}$ & $\begin{array}{c}27 \% \\
(19 / 70)\end{array}$ & $\begin{array}{c}7 \% \\
(5 / 70)\end{array}$ & $\begin{array}{c}7 \% \\
(5 / 70)\end{array}$ & $\begin{array}{l}100 \% \\
(2 / 2)\end{array}$ & - & - & - \\
\hline
\end{tabular}


des lexèmes et constructions. Ce problème concerne quatre des huit mots et constructions étudiés en détail dans le cadre de cet article, avec uniquement 2 occurrences pour chemise(s), 0 pour Monsieur, 3 pour tout le monde et 2 pour gouvernement en entretien. Il est donc indispensable de recueillir également des données élicitées afin d'obtenir des données comparables. Alors que les noms concrets peuvent être élicités par des images ( $c f$. section 2.1), des histoires illustrées ou les thématiques abordées par le guides d'entretien, des tâches de lecture sont incontournables pour les autres classes de mots et pour les constructions.

La lecture présente bien évidemment l'inconvénient d'être influencée par la forme graphique, comme nous l'avons montré pour les cas de semaine et tout le temps (construction figée décodée mot par mot). Cependant, cette influence de la graphie ne se limite pas au processus de décodage graphie-phonie en temps réel. Les formes graphiques sont en effet également stockées dans la mémoire lexicale, sous forme visuelle, ou encore sous forme phonologique à partir de la lecture à haute voix ( $c f$. aussi Andreassen et Lyche, $2018: 20$ ). Chez une partie de nos participants, nous avons ainsi observé, dans le cas de Monsieur, des prononciations avec [õ] également en répétition. L'influence de la forme graphique n'est donc pas seulement un biais méthodologique à éviter, mais aussi une réalité à prendre en compte dans l'étude des variétés d'apprenants à travers une triangulation de méthodes.

Par ailleurs, nos résultats montrent qu'il n'y a pas de différences systématiques chez les apprenants entre la lecture d'une liste de mots et celle d'un texte pour le phénomène étudié, vu que les résultats sont pratiquement identiques pour les deux tâches. Il s'agit là d'une différence par rapport au comportement en lecture des locuteurs L1, qui contrôlent leur prononciation davantage dans la lecture d'une liste de mots que dans celle d'un texte (cf. section 2.1). Dans les recherches sur la prononciation en FLE, la lecture d'une liste de mots et de constructions peut donc être une méthode prometteuse du point de vue de l'économie de la recherche, même si la graphie reste un facteur parasite. Il serait cependant important d'augmenter le nombre d'items à tester pour varier de façon plus systématique les contextes phonotactiques en proposant p. ex. semaine, la s(e)maine et une semaine (en lecture comme en répétition).

Pour ce qui est de la répétition, notre analyse des mots apparaissant en lecture, en parole spontanée et en répétition montre qu'il ne s'agit pas d'une tâche menant à des données plus valables que la lecture et qu'elle peut aussi créer des formes artificielles. Elle montre tout au moins que les apprenants sont capables de prononcer les mots en question avec ou sans schwa. Les exceptions à cette tendance sont particulièrement intéressantes : dans les cas de Monsieur, de tout le temps et de tout le monde, certains apprenants (de niveau intermédiaire dans le cas des constructions figées) ne reproduisent pas ce qu'ils entendent, mais leurs propres représentations erratiques. Celles-ci peuvent découler de la forme graphique ( $c f$. supra), mais aussi de leurs représentations cognitives provenant d'autres langues (p. ex. dans le cas des cognats comme fr. appartement/angl. apartment/all. Appartment).

Finalement, les résultats de notre analyse permettent de répondre de la manière suivante aux deux questions de recherche formulées dans la section 2.2 : 
1) De quelle manière la tâche (parole (semi-)spontanée en entretien guidé $v s$ parole élicitée dans différentes tâches avec et sans présentation de la forme graphique) influence-t-elle la production du schwa des apprenants autrichiens ? En répétition, les apprenants réalisent correctement et à un taux très élevé (entre $78 \%$ à 91\%) un schwa (avec l'exception de Monsieur : 53\%). En lecture, en revanche, la variante majoritaire dépend du contexte phonotactique et lexical : $[\mathrm{e}] /[\varepsilon]$ sont attestés en première syllabe, schwa dans le clitique le des constructions figées tout le temps et tout le monde, d'autres voyelles dans Monsieur et l'élision dans les cognates appartement et gouvernement. En parole spontanée, finalement, les résultats dépendent encore du contexte : alors que les réalisations se rapprochent davantage de la norme cible dans premier et tout le temps, avec un peu plus de schwa que de $[e] /[\varepsilon]$ pour le premier cas et l'élision dans le second, les cognates appartement et gouvernement restent à 100\% sans schwa.

2) La tâche de répétition en tant que méthode d'élicitation sans présentation de la forme graphique mène-t-elle à des données plus valables et comparables que la tâche de lecture? Comme l'élicitation par la lecture, la tâche de répétition permet d'obtenir des données comparables, notamment de mots peu fréquents dans la parole spontanée. Or, nous ne pouvons pas considérer les résultats comme plus valables car les locuteurs y montrent uniquement qu'ils sont capables de produire un son et non ce qu'ils produisent réellement dans le contexte phonotactique, dans le mot et dans la chaîne parlée.

La comparaison des tâches permet ainsi de conclure qu'une bonne partie des erreurs remonte au manque d'instruction explicite en phonétique et phonologie en classe de FLE : En effet, les élèves n'apprennent ni les correspondances graphiephonie, ni les règles phonologiques du français ni la prononciation des mots individuels. Ils acquièrent en revanche à partir de l'input phonique des enseignants et des matériaux sonores en classe ainsi que l'input graphique des manuels des représentations cognitives, qui se basent ainsi en partie sur des formes graphiques. De plus, ces représentations se construisent sur la base des cognats dans leur L1 et leur première langue étrangère, l'anglais.

\section{BIBLIOGRAPHIE}

Andreassen, H. N. et Lyche, C. (2018). Le rôle de la variation dans le développement phonologique : acquisition du schwa illustrée par deux corpus d'apprenants norvégiens. Synergies Pays Scandinaves, $13: 13-24$.

Boersma, P. et Weenink, D. (2018). Praat : doing phonetics by computer. http://www.fon.hum.uva.nl/praat/ [Computer program. Version 6.0.10].

Buben, V. (1935). Influence de l'orthographe sur la prononciation du français moderne. Bratislava : Filosofická fakulta University Komenského.

Chalier, M. (2018). Quelle norme de prononciation au Québec? Attitudes, représentations et perceptions. Langage et Société, 163(1) : 121-144.

Chalier, M. (2019). Les normes de prononciation du français : une étude perceptive panfrancophone. Thèse de doctorat, Université de Vienne.

Chervinski, J. (2013). Aussprachefehler von Münchner Lehramtsstudenten im Französischen - eine korpusbasierte Studie im Rahmen des Projekts IPFC. Mémoire de Master, Université de Munich. 
Conseil de l'Europe (dir.) (2018). Cadre européen commun de référence pour les langues. Apprendre enseigner - évaluer. Paris : Didier.

Côté, M.-H. (2012). Laurentian French (Québec) : Extra vowels, missing schwas and surprising liaison consonants. In : R. Gess, C. Lyche et T. Meisenburg (dir.), Phonological Variation in French : Illustrations from Three Continents. Amsterdam : John Benjamins, pp. 235-274.

Detey, S. et Le Gac, D. (2008). Didactique de l'oral et normes de prononciation : quid du français 'standard' dans une approche perceptive ? In : J. Durand, B. Habert et B. Laks (dir.), Congrès Mondial de Linguistique Française - CMLF'08. Paris : Institut de Linguistique Française, pp. 475-487.

Detey, S., Durand, J., Laks, B. et Lyche, C. (dir.) (2010). Les variétés du français parlé dans l'espace francophone. Ressources pour l'enseignement. Paris : Éditions Ophrys.

Detey, S., Durand, J., Laks, B. et Lyche, C. (dirs.) (2016). Varieties of Spoken French: a Source Book. With DVD. Oxford : Oxford University Press.

Detey, S., Lyche, C., Racine, I., Schwab, S. et Le Gac, D. (2016). The notion of norm in spoken French. In : S. Detey, J. Durand, B. Laks et C. Lyche (dir.). Varieties of Spoken French: a source book. With DVD. Oxford : Oxford University Press, pp. 55-67.

Dewaele, J.-M. (2004). The acquisition of sociolinguistic competence in French as a foreign language: An overview. Journal of French Language Studies, $14: 301-319$.

Durand, J., Laks, B. et Lyche, C. (2002). La phonologie du français contemporain : usages, variétés et structure. In : C. Pusch et W. Raible (dir.), Romanistische Korpuslinguistik - Korpora und gesprochene Sprache/Romance Corpus Linguistics - Corpora and Spoken Language. Tübingen : Narr, pp. 93-106.

Durand, J., Laks, B. et Lyche, C. (dirs.) (2009). Phonologie, variation et accents du français. Paris : Hermès.

Flege, J. (1987). The production of 'new' and 'similar' phones in a foreign language: evidence for the effect of equivalence classification. Journal of Phonetics, $15: 47-65$.

Fouché, P. (1959). Traité de prononciation française. Paris : Klincksieck.

Fougeron, C., Gendrot, C. et Bürki, A. (2007). On the acoustic characteristics of French schwa. ICPHS Saarbrücken : 941-944.

Hansen, A. B. (1994). Etude du E caduc - stabilisation en cours et variations lexicales. Journal of French Language Studies, 4(1): 25-54.

Isely, R., Racine, I., Detey, S. et Andreassen, H. (2018). Le rôle de l'immersion dans l'apprentissage du schwa chez les apprenants alémaniques avancés de FLE. Congrès Mondial de Linguistique Française - CMLF'18.

Labov, W. (1972). Sociolinguistic Patterns. Oxford : Blackwell.

Léon, P. (2011). Phonétisme et prononciations du français. Paris : Armand Colin.

Luner, C., Truxa-Pirierros E. et Wladika, E. (2014). Bien fait! Band 1 für die Unterstufe, Lehrbuch. Vienne : Verlag Hölter-Pichler-Tempsky.

Luner, C. et Berchotteau, C. (2015). Bien fait!, Band 2, Lehrbuch. Vienne : Verlag Hölter-Pichler-Tempsky.

Luner, C. et Berchotteau, C. (2017). Bien fait!, Band 3, Lehrbuch. Vienne : Verlag Hölter-Pichler-Tempsky.

Lyche, C. (2010). Le français de référence : éléments de synthèse. In : S. Detey, J. Durand, B. Laks et C. Lyche (dir.), Les variétés du français parlé dans l'espace francophone: ressources pour l'enseignement. Paris : Ophrys, pp. 143-165.

Lyche, C. (2016). Approaching variation in PFC. In : S. Detey, J. Durand, B. Laks et C. Lyche (dirs.), Varieties of Spoken French : a Source Book. With DVD. Oxford : Oxford University Press, pp. 252-262.

Lyche, C. et Østby, K. (2009). Le français de la haute bourgeoisie parisienne : une variété conservatrice? In : J. Durand, B. Laks et C. Lyche (dirs.) : Phonologie, variation et accents du français. Paris : Hermès, pp. 203-230.

Morin, Y.-C. (2000). Le français de référence et la norme de prononciation. In : M. Francard, G. Geron et R. Wilmet (dir.) : Actes du colloque de Louvain-la-Neuve 3-5 novembre 1999, Cahiers de l'Institut de Linguistique de Louvain 26, pp. 91-135.

Moyer, A. (2007). Empirical considerations on the age factor in L2 phonology. Issues in Applied Linguistics, 15(2) : 109-127.

Munro, M. et Derwing, T. (2015). A prospectus for pronunciation research in the 21 st century. Journal of Second Language Pronunciation, 1(1) : 11-42.

Pustka, E., Forster, J. et Kamerhuber, J. (2018). Failures in vocabulary learning instead of pronunciation difficulties: schwa and liaison in learner French. In: M. Belz, C. Mooshammer, S. Fuchs, S. Jannedy, 
O. Rasskazova and M. Żygis (dir.), Proceedings of the Conference on Phonetics \& Phonology in Germanspeaking Countries (P\&P 13). Berlin: Humboldt-Universität zu Berlin, pp. 153-156.

Pustka, E. et Meisenburg, T. (2017). Les germanophones. In : S. Detey, I. Racine, Y. Kawaguchi et J. Eychenne (dir.), La prononciation du français dans le monde : du natif à l'apprenant. Paris : CLE International, pp. 130-136.

Pustka, E. (2007). Phonologie et variétés en contact : Aveyronnais et Guadeloupéens à Paris. Tübingen : Narr.

Racine, I. et Detey, S. (2019). Production of French close rounded vowels by Spanish learners : a corpusbased study. In : M. Gibson et J. Gil (dir.), Romance Phonetics and Phonology. Oxford : Oxford University Press, pp. 381-394.

Racine, I., Detey, S., Zay, F. et Kawaguchi, Y. (2012). Des atouts d'un corpus multitâches pour l'étude de la phonologie en L2 : l'exemple du projet 'Interphonologie du français contemporain' (IPFC). In : A. Kamber et C. Skupiens (dir.), Recherches récentes en FLE. Berne : Peter Lang, pp. 1-19.

Shea, C. (2013). Methods for researching second language phonological and phonetic acquisition. Studies in Hispanic and Lusophone Linguistics, 6 : 179-196.

Thibault, A (2012). Les avatars du schwa colonial dans le créole des Petites Antilles. In : A. Thibault (dir.). Le français dans les Antilles. Études linguistiques. Paris : L'Harmattan, pp. 243-269.

Thomas, A. (2001). Schwa au niveau avancé du français langue seconde. Revue Canadienne de Linguistique Appliquée, 4 : 103-112.

Thomas, A. (2002). La variation phonétique en français langue seconde au niveau universitaire avancé. Acquisition et Interaction en Langue Etrangère, 17 : 101-121.

Thomas, A. (2004). Phonetic norm versus usage in advanced French. IRAL, $42: 365-382$.

Uritescu, D., Mougeon, R. et Handouleh, Y. (2002). Le comportement du schwa dans le français parlé par les élèves des programmes d'immersion française. La linguistique fonctionnelle au tournant du siècle. In : A. Baudot et C. Tatilon (dir.), Actes du Vingt-quatrième Colloque international de linguistique fonctionnelle. Toronto : Editions du GREF, pp. 335-346.

Uritescu, D., Mougeon, R., Rehner, K. et Nadasdi, T. (2004). Acquisition of the internal and external constraints of variable schwa deletion by French immersion students. IRAL, $42: 349-364$.

Wlasak-Feik, C., Winz, P., Buschhaus, M. et Mann-Grabowski, C. (2016). Parcours plus. Berlin : Cornelsen.

\section{ANNEXE}

Protocole de l'entretien guidé:

Généralités :

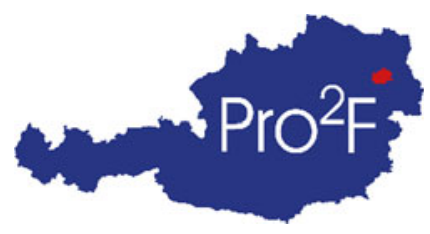

Quel âge as-tu ?*

Quelle est ta nationalité ?

École :

Décris ta journée typique!

Qu'est-ce que tu vois dans cette pièce (autour de nous)?

De quelle heure à quelle heure tu as cours?

Qu'est-ce qui tu aimes à l'école ? Quelle est ta matière préférée ? Pourquoi ?

Qu'est-ce que tu n'aimes pas à l'école ?

Environnement :

Qu'est-ce que tu aimes à Vienne ?

Décris le chemin pour aller de chez toi à la gare la plus proche. 
Fêtes :

Qu'est-ce que tu fais à ton anniversaire ? (famille/copains ?)

Tu fêtes Noël comment?

Loisirs :

Qu'est-ce que tu aimes faire pendant ton temps libre ? (musique, cinéma, sport etc.)

Quel est ton film préféré?

Qu'est-ce que tu aimes manger?

Qu'est-ce que tu aimes faire pendant les vacances?

Tu t'habilles comment?

Qu'est-ce que tu as fait hier?

Amis/Famille :

Où est-ce que tu aimes rencontrer tes amis ? (école, sport, café ?)

Décris ton meilleur ami !

Raconte-moi quelque chose sur ta famille!

Voyages :

As-tu déjà voyagé dans un pays étranger ? Si oui, raconte ton/tes voyage(s).

Si non, quels pays aimerais-tu visiter et pourquoi?

Es-tu déjà allé en France ou dans un autre pays francophone?

Quel pays francophone tu aimerais visiter ? Pourquoi ?

Quel est ton pays préféré ? Pourquoi ?

Projets :

Quels sont tes projets pour le futur (professionnels, personnels, etc.) ?

Langues/Français :

Quelles langues parles-tu ?

Quand as-tu commencé à étudier le français?

Où as-tu commencé à étudier le français? (lycée, école primaire ?)

Pourquoi as-tu choisi d'étudier le français?

Qu'est-ce qui te paraît difficile en français ? Qu'est-ce que tu n'arrives pas à faire ?

À quoi est-ce que tu penses quand tu entends le mot France?

${ }^{*}=$ questions obligatoires en gras

Cite this article: Kamerhuber J, Horvath J et Pustka E (2020). Lecture, répétition, parole spontanée : l'impact de la tâche sur le comportement du schwa en FLE. Journal of French Language Studies 30, 161-188. https:// doi.org/10.1017/S095926952000006X 